\title{
Algebraic Structures of Characteristics in Involutive Systems of Non-Linear Partial Differential Equations
}

\author{
By \\ Kunio KAKIÉ*
}

\section{Introduction}

An involutive system of partial differential equations is, roughly speaking, such a system that its general solution can be obtained by solving successively equations of Cauchy-Kowalevsky's type. It was E. Cartan who introduced the notion of involution for exterior differential systems. On this subject one has the Cartan-Kähler theorem and the prolongation theorem due to E. Cartan, M. Kuranishi and M. Matsuda (cf. Cartan [2, 4]; Kuranishi [11-12]; Matsuda [13-14]). M. Kuranishi constructed the process of standard prolongation, which was applied to infinite Lie groups by himself. It was also applied to the equivalence problem of G-structures by V.W. Guillemin, I.M. Singer and S. Sternberg. In this course they clarified the algebraic structures of inrolutive systems. By their results $M$. Kuranishi gave a clear definition of involutive systems. His standard prolongation was improved by M. Matsuda, who combined it with the classical method of prolongation due to Lagrange and Jacobi.

On the other hand, it is well-known that, in the classical and modern theory of partial differential equations, consideration of characteristics in various senses leads us to fruitful results. In our subject the two concepts of Cauchy characteristics and Monge ones will be particularly important. Effectiveness of the former was shown by E. Cartan for general systems ([2-4]). However, that of the latter seems to have been shown only for special systems (cf. Cartan [4], Chap. IV, Part III).

The principal aim of this paper is to investigate algebraic structures

Communicated by M. Sato, June 10, 1976.

* Department of Mathematics, Rikkyo University, Nishi-Ikebukuro, Tokyo 171, Japan. 
of the above two characteristics of general non-linear involutive systems. In our investigation, a principal role will be played by the introduced concept "characteristic module". It is to be emphasized that we deal with non-linear involutive systems with several unknown functions and that we discuss also the case when the characters of higher order do not necessarily vanish. Besides, our investigation enables us to establish the method of integration for involutive systems whose characters of order more than one vanish, generalizing the one obtained recently by the present author ([9-10]).

All notions such as functions and manifolds occurring in this paper are assumed to be in the real or complex analytic category though our argument can be carried out in the $C^{\infty}$ category except when the CartanKähler existence theorem is applied.

Our main results are summarized as follows. Let $\Phi$ be a system of partial differential equations of order $l$ in a fibered manifold $(\mathcal{M}, \mathcal{N}, \rho)$. It is defined to be a locally finitely generated subsheaf of ideals in the sheaf of rings of germs of analytic functions on the space of $l$-jets of cross-sections of $(\mathscr{M}, \mathcal{N}, \rho)$. Suppose that $\Phi$ is involutive. We introduce the characteristic module $M$ of $\Phi$ by generalizing the characteristic ideal defined for systems with a single unknown function (cf. Kakie [10]); The latter is, roughly speaking, the ideal in a polynomial ring generated by the characteristic polynomials of all differential equations in $\Phi$. The module $M$ is a homogeneous submodule of that Noetherian graded module $L$ over a polynomial ring which is canonically associated with $(\mathcal{M}, \mathcal{\Omega}, \rho)$. The characteristic module $M$ admits a reduced primary decomposition in $L: M=\bigcap_{j=1}^{\nu} Q_{j}$ where each $Q_{j}$ is a primary homogeneous submodule of $L$. For a homogeneous submodule $N$ of $L$, we denote by $\mu(M ; L)$ the multiplicity of $N$ with respect to $L ; \mu(M ; L)$ is defined to be the positive integer obtained from the coefficient of the highest degree $r$ of the Hilbert characteristic polynomial of the graded module $L / N$ by multiplying it by $r$ !. Let $s_{1}, s_{2}, \cdots, s_{n}$ be the Cartan's characters of $\Phi$ of order $1,2, \cdots, n$ respectively $(n=\operatorname{dim} \Omega)$. One of the fundamental results is the following: Assume that $\Phi$ is involutive and that $s_{p}>0, s_{p+1}=\cdots=s_{n}=0$. Let $\mathfrak{P}_{j}$ be the associated prime ideal of $Q_{j}(1 \leqq j \leqq \nu)$. Then the greatest projective dimension of the homogeneous prime ideals $\mathfrak{P}_{j}$ is equal to $p-1$. 
Moreover the following is valid:

$$
s_{p}=\sum_{\text {proj dim } \Re_{j}=p-1} \mu\left(Q_{j} ; L\right)
$$

(Theorem III in $\S 5$ ). The primary decomposition of $M$ enables us to investigate algebraic structures of Monge characteristics of $\Phi$. Corresponding to each $\mathfrak{P}_{j}$, a Monge characteristic system of $\bar{\Phi}$ is defined (§§6-7). Furthermore we can give a sufficient condition in order that the differential system associated with $\Phi$ admits Cauchy-Cartan characteristics (Theorem IV). Suppose that $s_{2}=\cdots=s_{n}=0$. In this case, we can clarify more completely algebraic structures of Monge characteristics. In fact, considering the problem of constructing from $\Phi$ suitable involutive systems for integration, we can show effectiveness of Monge characteristics (Theorems V and VI in §9). It is a classical problem to find a process of reducing the solution of the Cauchy problem for $\Phi$ with a given initial value to the integration of a completely integrable Pfaffian system. As an application of our theory, we can solve this problem for $\Phi$ with $s_{2}=\cdots=s_{n}=0$ (cf. Kakié [9-10]).

This paper is divided into three parts. Part I (§§1-3) is devoted to the investigation on modules associated with involutive subspaces. Part II ( $\S 4_{4-7)}$ is concerned with involutive systems of partial differential equations. The concept of characteristic module is introduced and algebraic structures of Monge characteristics is studied. In part III (§§ 8-10), we deal with only involutive systems of which characters of order more than one vanish.

The author would like to express his sincere gratitude to Professor M. Sato for his perspicacious suggestion and encouragement during the investigation. It is orving to his suggestion that the algebraic discussion becomes much simpler than the original one.

The author also would like to express his sincere gratitude to Professor M. Kuranishi for his kind advice and encouragement.

\section{Part I. Modules Associated with Involutive Subspaces}

\section{§ 1. Primary Decomposition}

Let $K$ be a field of characteristic zero, and $R$ be the polynomial 
ring over $K$ in $n$ variables. The ring $R$ is a graded one with usual direct sum decomposition: $R=\sum_{q=0}^{\infty} R_{q}$. Let $L=\sum_{q=0}^{\infty} L_{q}$ be a finitely generated graded $R$-module. Obviously $L$ is a Noetherian module; it satisfies the maximal condition concerning its submodules. Let $M=\sum_{q=0}^{\infty} M_{q}$ be a homogeneous $R$-submodule of $L$. The submodule $M$ admits a reduced primary decomposition in $L: M=\bigcap_{j=1}^{\nu} Q_{j}$ where the modules $Q_{j}$ are homogeneous primary submodules of $L$ (cf. Bourbaki [1], Chap. IV). Let $Q_{j}$ be $\Re_{j}$-primary in $L$. For any element $\xi$ in $R$, the homomorphism $h_{\xi}$ in the $R$-module $L / Q_{j}$ defined by $h_{\xi}(z)=\xi z$ is injective or locally nilpotent (presque nilpotent). The ideal $\mathfrak{P}_{j}$ is the set of all elements $\xi \in R$ such that the homomorphism $h_{\xi}$ is locally nilpotent. Since $M$ is a homogeneous submodule, the ideals $\mathfrak{F}_{j}$ are necessarily homogeneous. The reduced primary decomposition is unique in the sense that the number $\nu$ of the submodules $Q_{j}$ and the set $\left\{\mathfrak{F}_{1}, \cdots, \mathfrak{P}_{\nu}\right\}$ are uniquely determined only by $M$. Let $\operatorname{Ass}(N)$ denote the set of prime ideals associated with an $R$-module $N$. Then Ass $(L / M)=\left\{\mathfrak{P}_{1}, \cdots, \mathfrak{P}_{\nu}\right\}$. Moreover if $\mathfrak{P}_{j}$ is a minimal element in the set Ass $(L / M)$, the corresponding submodule $Q_{j}$ is uniquely determined by $M$.

Let $P(N, x)$ denote the characteristic polynomial of Hilbert of $N$, where $N=\sum_{q=0}^{\infty} N_{q}$ is a finitely generated graded $R$-module. (cf. Serre [17], Chap. II; Zeriski-Samuel [18], Chap. VII, §12). The number $P(N, q)$ indicates $\operatorname{dim} N_{q}$ for every sufficiently large integer $q$.

Let $r$ be the degree of $P(L / M, x)$. The polynomial $P(L / M, x)$ can be written in the following form:

$$
P(L / M, x)=a_{0}\left(\begin{array}{l}
x \\
r
\end{array}\right)+a_{1}\left(\begin{array}{c}
x \\
r-1
\end{array}\right)+\cdots+a_{r},
$$

where $\left(\begin{array}{l}x \\ s\end{array}\right)=\frac{x(x-1) \cdots(x-s+1)}{s !}$ and the coefficients $a_{0}, \cdots, a_{r}$ are integers. The coefficient $a_{0}$ is usually called the multiplicity of $M$ with respect to $L$ over $K$. We shall denote it by $\mu(M ; L) ; \mu(M ; L)=a_{0}$. It is a positive integer except the case when $M_{q}=L_{q}$ for large $q$.

The degree of $P(L / M, x)$ is closely related to the projective dimensions of the homogeneous ideals in $\operatorname{Ass}(L / M)$. In fact, the following is valid (Zariski-Samuel [18], p. 235, Theorem 42'). 
Lemma 1. The degree of the characteristic polynomial $P(L / M$, $x)$ is equal to the greatest projective dimension of the associated prime ideals Ass $(L / M)$ of $L / M$.

Let $\mathcal{G}_{p}$ be the set of all $p$-dimensional subspaces of the vector space $R_{1}$ of dimension $n$ over $K(0 \leqq p \leqq n)$. $\mathscr{L}_{p}$ forms a $(n-p) p$-dimensional algebraic variety with Plücker coordinates. This is usually called Grassmann manifold when $K$ is the field of real or complex numbers.

The multiplicity $\mu(M ; L)$ possesses a geometrical interpretation. In fact, one has the following

Lemma 2. Let $r$ be the degree of $P(L / M, x)$. Then the following are valid:

(i) Let $\rho$ be the smallest integer $p$ for which there is an element $F_{p} \in \mathcal{G}_{p}$ such that $\left(M+F_{p} L\right) \cap L_{q}$ coincides with $L_{q}$ for all large $q$. Then $r$ is equal to $\rho-1$.

(ii) For any element $F_{r} \in \mathcal{L}_{r}$ outside its proper algebraic subvariety, the characteristic polynomial of the graded $R$-module $L /(M$ $\left.+F_{r} L\right)$ is of degree 0 and $\mu(M ; L)$ is equal to $\mu\left(M+F_{r} L ; L\right)$. Moreover

$$
\mu(M ; L)=\min \left\{\operatorname{dim} L_{q} /\left(M+F_{r} L\right) \cap L_{q} ; F_{r} \in \mathcal{G}_{r}\right\}
$$

for sufficiently large $q$.

Proof. We prove this lemma by dividing into five steps. In this proof, we denote $\mu(M ; L)$ simply by $\mu(M)$. By polynomials of negative degrees we mean zero.

(a) Let $F_{p}$ be an arbitrary element of $\mathcal{G}_{p}(0 \leqq p \leqq n)$. Then the characteristic polynomial of $L /\left(M+F_{\rho} L\right)$ is of degree $\geqq r-p$. If $F_{r}$ is an element of $\mathscr{G}_{r}$ such that the characteristic polynomial of $L /\left(M+F_{r} L\right)$ is exactly of degree 0 , then,$\prime(M)$ is equal to or less than $\mu\left(M+F_{r} L\right)$.

Proof. We write $N=L / M$. Let us consider the homomorphism $\theta_{\eta}$ from $N$ into itself defined by $\theta_{\eta}(y)=\eta y$, where $\eta \in R_{1}$. Writing $S=\operatorname{ker} \theta_{\eta}$ and $T=N / \theta_{\eta}(N)$, we have the exact sequence of modules

$$
0 \rightarrow S \stackrel{i}{\rightarrow} N \stackrel{\theta_{\eta}}{\rightarrow} N \stackrel{j}{\rightarrow} T \rightarrow 0
$$


where $i$ and $j$ are natural homomorphisms. This gives rise to the exact sequence of vector spaces over $K$

$$
0 \rightarrow S_{q} \stackrel{i}{\rightarrow} N_{q} \stackrel{\theta_{\eta}}{\rightarrow} N_{q+1} \stackrel{j}{\rightarrow} T_{q+1} \rightarrow 0
$$

where $N=\sum_{q=0}^{\infty} N_{q}, S=\sum_{q=0}^{\infty} S_{q}, T=\sum_{q=0}^{\infty} T_{q}$ (direct sum decompositions of graded modules). Obviously

$$
T_{q+1}=N_{q+1} / \theta_{\eta}\left(N_{q}\right)=L_{q+1} /(M+\eta L) \cap L_{q+1} .
$$

Hence, applying the well-known lemma (Zariski-Samuel [18], p. 233, Lemma), we get from the above exact sequence the following equalities:

$$
P(N, q+1)-P(N, q)=P(L /(M+\eta L), q+1)-\operatorname{dim} S_{q}
$$

( $q$ : sufficiently large).

The term on the left side can be written in the form

$$
\begin{gathered}
P(N, q+1)-P(N, q)=\mu(M)\left(\begin{array}{c}
q \\
r-1
\end{array}\right)+b_{1}\left(\begin{array}{c}
q \\
r-2
\end{array}\right)+\cdots+b_{r-1} \\
=\mu(M)\left(\begin{array}{l}
q+1 \\
r-1
\end{array}\right)+c_{1}\left(\begin{array}{c}
q+1 \\
r-2
\end{array}\right)+\cdots+c_{r-1} .
\end{gathered}
$$

Therefore we conclude from (1) that the characteristic polynomial of $L /(M+\eta L)$ is of degree $\geqq r-1$, and that if it is of degree $r-1, \mu(M)$ $\leqq \mu(M+\eta L)$. Proceeding step by step in just the same manner, we know that the characteristic polynomial of $L /\left\{M+\left(\eta_{1}, \cdots, \eta_{p}\right) L\right\}$ is of degree $\geqq r-p(1 \leqq p \leqq n)$, and that if the characteristic polynomial of $L /\{M$ $\left.+\left(\eta_{1}, \cdots, \eta_{r}\right) L\right\}$ is exactly of degree 0 , then that of $L /\left\{M+\left(\eta_{1}, \cdots, \eta_{p}\right) L\right\}$ is of degree $r-p$ for each $p=1,2, \cdots, r$. In this case it also holds that

$$
\mu(M) \leqq \mu\left(M+\eta_{1} L\right) \leqq \cdots \leqq \mu\left(M+\left(\eta_{1}, \cdots, \eta_{r}\right) L\right)
$$

where $\eta_{p} \in R_{1}$. this completes the proof of (a).

(b) There exists a sequence $F_{0} \subset \cdots \subset F_{r} \subset F_{r+1}$ where $F_{p} \in \mathcal{G}_{p}$ such that the characteristic polynomial of $L /\left(M+F_{p} L\right)$ is exactly of degree $r-p(0 \leqq p \leqq r+1)$, and that $\mu(M)=\mu\left(M+F_{1} L\right)=\cdots=\mu\left(M+F_{r} L\right)$.

Proof. We use the same notations as in the proof of (a). Suppose firstly that none of the ideals in $\operatorname{Ass}(N)=\left\{\mathfrak{P}_{1}, \cdots, \mathfrak{P}_{\nu}\right\}$ coincides with the maximal ideal $\mathfrak{X}=\sum_{q=1}^{\infty} R_{q}$ in $R$. We can choose an element $\eta_{1} \in R_{1}$ 
not belonging to any ideal $\mathfrak{S}_{j}$. Then the homomorphism $\theta_{\eta_{1}}$ is injective (cf. Bourbaki [1], p. 132, Corollaire 2). Hence ker $\theta_{\eta_{1}}=0$. Suppose secondly that one of the ideals $\mathfrak{F}_{j}$, say $\mathfrak{P}_{\nu}$, coincides with $\mathfrak{X}$. Since $\mathbb{Z}^{\sigma} L \subset Q_{\nu}$ for some positive integer $\sigma$, it follows that $Q_{\nu} \cap L_{q}=L_{q}$ for large $q$. Therefore $M_{q}=\bigcap_{\substack{j=1 \\ \nu-1}}^{\nu-1} Q_{j} \cap L_{q}$ for large $q$. Let $\eta_{1}$ be an element in $R_{1}$ not belonging to $\bigcup_{j=1}^{\nu-1} \mathfrak{P}_{j}$. By the same reason as above, $\theta_{\eta_{1}}$ is injective in $N_{q}$, and hence $\operatorname{ker} \theta_{\eta_{1}} \cap N_{q}=0$ for large $q$. Hence, in any case, we have the equality (1) in which $\eta=\eta_{1}$ and $\operatorname{dim} S_{q}=0$. By the remark below (1), it follows that $P\left(L /\left(M+\eta_{1} L\right), x\right)$ is of degree $r-1$ and that $\mu\left(M+\eta_{1} L\right)=\mu(M)$. Repeating the same argument successively, we can find $r+1$ elements $\eta_{1}, \cdots, \eta_{r+1}$ in $R_{1}$ such that the characteristic polynomial of $L /\left\{M+\left(\eta_{1}, \cdots, \eta_{p}\right) L\right\}$ is of degree $r-p$ for each $p=1,2, \cdots, r+1$, and that $\mu(M)=\mu\left(M+\eta_{1} L\right)=\cdots=\mu\left(M+\left(\eta_{1}, \cdots, \eta_{r}\right) L\right)$. As is readily seen, $\eta_{1}, \cdots, \eta_{r+1}$ are necessarily linearly independent. Let $F_{p}$ be the space spanned by $\eta_{1}, \cdots, \eta_{p}$. Then the space $F_{p}$ have the required property.

(c) There is an element $F_{r+1}$ of $\mathcal{L}_{r+1}$ such that

$$
\left(M+F_{r+1} L\right) \cap L_{q}=L_{q} \text { for large } q .
$$

Proof. This is an immediate consequence of (b).

(d) Let $N^{\prime}=\sum_{q=0}^{\infty} N_{q}^{\prime}$ be a homogeneous submodule of $L$ such that there is an element $\eta \in R_{1}$ for which $\left(N^{\prime}+\eta L\right) \cap L_{q}=L_{q}$ for large $q$. Then $\operatorname{dim} \mathrm{L}_{q} / N_{q}^{\prime} \geqq \operatorname{dim} L_{q+1} / N_{q+1}^{\prime}$ for large $q$.

Proof. The assumption implies that the homomorphism defined by the multiplication by $\eta$ from $L_{q} / N_{q}^{\prime}$ to $L_{q+1} / N_{q+1}^{\prime}$ is surjective for large $q$. The required result follows immediately from this.

(e) We are now in a position to complete the proof of Lemma 2. If there is an element $F_{d+1} \in \mathcal{G}_{d+1}$ such that $\left(M+F_{d+1} L\right) \cap L_{q}=L_{q}$ for large $q$, then $P(L / M, x)$ is of degree $\leqq d$. In fact, recalling that $P(L / M, q)$ $=\operatorname{dim} L_{q} / M_{q}$ for large $q$, we find no difficulty in showing that

$$
P(L / M, q) \leqq \text { Const. }\left(\begin{array}{c}
q+d \\
q
\end{array}\right) \text { for large } q .
$$

This inequality implies that $P(L / M, x)$ is of degree $\leqq d$. It follows from this and (c) that the assertion (i) is valid.

Let us next prove (ii). Since (c) is valid, we can readily prove 
that the set $S$ of elements $F_{r} \in \mathcal{Q}_{r}$ for which there is no element $\eta \in R_{1}$ such that (2) holds for the space $F_{r+1}$ spanned by $F_{r}$ and $\eta$ forms a proper algebraic subvariety of $\mathscr{L}_{r}$. We put

$$
\begin{aligned}
& \delta_{q}\left(F_{r}\right)=\operatorname{dim} L_{q} /\left(M+F_{r} L\right) \cap L_{q}, \\
& \delta_{q}=\min \left\{\delta_{q}\left(F_{r}\right) ; F_{r} \in \mathcal{G}_{r}\right\} .
\end{aligned}
$$

The function $\delta_{q}\left(F_{r}\right)$ attains its minimum value outside a proper algebraic subvariety $D(q)$ of $\mathscr{G}_{r}$. By virtue of (b), there exists $F_{r} \in \mathcal{G}_{r}$ such that $\mu(M)=\mu\left(M+F_{r} L\right)=\delta_{q}\left(F_{r}\right)$ for large $q$. Hence we have $\delta_{q} \leqq$ $\mu(M)$ for large $q$. Let $F_{r}$ be an element of $\mathcal{L}_{r}$ not belonging to its proper subvariety $S \cup D(q)$. Since the assumption (d) is satisfied for the module $M+F_{r} L$, we have $\delta_{q}\left(F_{r}\right) \geqq \delta_{q+1}\left(F_{r}\right)$ if $q$ is large enough. It follows that

$$
\delta_{q}=\delta_{q}\left(F_{r}\right) \geqq \delta_{q+1}\left(F_{r}\right) \geqq \delta_{q+1}
$$

Thus the sequence $\left\{\delta_{q}\right\}$ of non-negative integers is a descending one when $q$ varies sufficiently large integers. We conclude from this fact that $\delta_{q}=\delta_{q+1}=\delta_{q+2}=\cdots$ for large $q$. Then it follows from (3) that for any $F_{r}$ outside $S \cup D(q), \delta_{q}\left(F_{r}\right)=\delta_{q+1}\left(F_{r}\right)=\delta_{q+1}\left(F_{r}\right)=\cdots$. From this it follows that the characteristic polynomial of $M+F_{r} L$ is of degree 0 and $\mu\left(M+F_{r} L\right)=\delta_{q}\left(F_{r}\right)=\delta_{q}$ for such $F_{r}$. On the other hand, (a) asserts that $\mu(M) \leqq \mu\left(M+F_{r} L\right)=\delta_{q}$ for such $F_{r}$. Combining this with the fact $\delta_{q} \leqq \mu(M)$ shown already, we have $\mu(M)=\delta_{q}$ for large $q$. Thus we have all the required results in (ii).

Q.E.D.

Let us now consider a reduced primary decomposition $M=\bigcap_{j=1}^{\nu} Q_{j}$ in $L, Q_{j}$ being $\mathfrak{P}_{j}$-primary. If the projective dimension of $\mathfrak{P}_{k}$ is equal to $\operatorname{deg} P(L / M, x)$, then $\mathfrak{F}_{k}$ is a minimal element in the set $\left\{\mathfrak{F}_{1}, \cdots, \mathfrak{F}_{\nu}\right\}$ (cf. Lemma 1), and hence the corresponding module $Q_{k}$ is uniquely determined only by $M$ by the uniqueness theorem of reduced primary decompositions: In particular, $\mu\left(Q_{k} ; L\right)$ is also so. There exists a link between the multiplicity $\mu(M ; L)$ and the multiplicities $\mu\left(Q_{j} ; L\right)$. In lact, the following theorem is valid. 
Theorem I. Let $M=\bigcap_{j=1}^{\nu} Q_{j}$ be a reduced primary decomposition of $M$ in $L$, and let $Q_{j}$ be $\mathfrak{P}_{j}$-primary. Let $r$ denote the degree of the Hilbert characteristic polynomial $P(L / M, x)$. Then

$$
\mu(M ; L)=\sum_{\text {proj dim } \Re_{j=r}} \mu\left(Q_{j} ; L\right) .
$$

To prove this theorem, we shall recall the following lemma.

Lemma 3. Let $N_{1}$ and $N_{2}$ be homogeneous submodules of $L$. Then

$$
P\left(L / N_{1} \cap N_{2}, x\right)+P\left(L /\left(N_{1}+N_{2}\right), x\right)=P\left(L / N_{1}, x\right)+P\left(L / N_{2}, x\right) .
$$

Proof. Obviously we have the following two exact sequences of $R$-modules with natural homomorphisms:

$$
\begin{aligned}
& 0 \rightarrow\left(N_{1}+N_{2}\right) / N_{1} \rightarrow L / N_{1} \rightarrow L /\left(N_{1}+N_{2}\right) \rightarrow 0, \\
& 0 \rightarrow N_{2} / N_{1} \cap N_{2} \rightarrow L / N_{1} \cap N_{2} \rightarrow L / N_{2} \rightarrow 0 .
\end{aligned}
$$

It follows from these that the following equalities are valid for all sufficiently large $q$ :

$$
\begin{aligned}
& P\left(\left(N_{1}+N_{2}\right) / N_{1}, q\right)-P\left(L / N_{1}, q\right)+P\left(L /\left(N_{1}+N_{2}\right), q\right)=0, \\
& P\left(N_{2} / N_{1} \cap N_{2}, q\right)-P\left(L / N_{1} \cap N_{2}, q\right)+P\left(L / N_{2}, q\right)=0 .
\end{aligned}
$$

$\left(N_{1}+N_{2}\right) / N_{1}$ is isomorphic to $N_{2} / N_{1} \cap N_{2}$, and hence we have the desired result.

Q.E.D.

Let $\mathfrak{x}_{L}(M)$ be the ideal in $R$ defined to be the set of all elements $\xi \in R$ such that $\xi^{\sigma} L \subset M$ for some integer $\sigma$. This ideal $\mathfrak{x}_{L}(M)$ coincides with the intersection of the prime ideals associated with $L / M ; \mathfrak{r}_{L}(M)$ $=\bigcap_{j=1}^{\nu} \mathfrak{P}_{j}$. It is clear that $\mathfrak{x}_{L}\left(N_{1}+N_{2}\right)$ contains $\mathfrak{r}_{L}\left(N_{1}\right)+\mathfrak{r}_{L}\left(N_{2}\right)$ for any two submodules $N_{1}$ and $N_{2}$ of $L$. we have

Proof of Theorem I: Applying Lemma 3 to $N_{1}=Q_{1}$ and $N_{2}=\bigcap_{j=2}^{\nu} Q_{j}$, 


$$
\begin{aligned}
P(L / M, x) & =P\left(L / \bigcap_{j=1}^{\nu} Q_{j}, x\right) \\
& =P\left(L / Q_{1}, x\right)+P\left(L / \bigcap_{j=2}^{\nu} Q_{j}, x\right)-P(L / N, x),
\end{aligned}
$$

where we write $N=Q_{1}+\bigcap_{j=2}^{\nu} Q_{j}$. Let us prove that the prime ideals associated with $L / N$ are of projective dimension less than $r$. Let $\mathfrak{A}$ be any element of $\operatorname{Ass}(L / N)$. Since $\mathfrak{r}_{L}(N)$ contains $\mathfrak{B}_{1}+\bigcap_{j=2}^{\nu} \mathfrak{P}_{j}$, the ideal $\mathfrak{U}$ also contains the latter ideal. It follows that $\mathfrak{A}$ contains $\mathfrak{P}_{1}$ and $\mathfrak{P}_{k}$ for some $k \neq 1$. If one of $\mathfrak{P}_{1}$ and $\mathfrak{P}_{k}$ is of projective dimension $<r$, then it is obvious that $\mathfrak{P}$ is also so. Assume that both ideals $\mathfrak{P}_{1}$ and $\mathfrak{P}_{k}$ are of projective dimension $r$. Then $\mathfrak{P}_{1}+\mathfrak{P}_{k}$ is distinct from $\mathfrak{P}_{1}$, for $\mathfrak{P}_{1} \neq \mathfrak{P}_{k}$. We conclude that $\mathfrak{A} \supsetneqq \mathfrak{P}_{1}$. Hence $\mathfrak{N}$ is of projective dimension $<r=$ proj $\operatorname{dim} \mathfrak{P}_{1}$, for in the contrary case, both prime ideals would have the same dimension and one of the two ideals contains the other, and hence they would coincide. Thus we have the desired result. What we have just deduced implies, by virtue of Lemma 1 , that $P(L / N$, $x)$ is of degree $<r$. Consequently the above formula indicates that the term of (the highest) degree $r$ of $P(L / M, x)$ is equal to the sum of those of $P\left(L / Q_{1}, x\right)$ and $P\left(L \bigcap_{j=2}^{\nu} Q_{j}, x\right)$; that is,

$$
P(L / M, x) \equiv P\left(L / Q_{1}, x\right)+P\left(L / \bigcap_{j=2}^{\nu} Q_{j}, x\right)\left(\bmod 1, x, \cdots, x^{r-1}\right) .
$$

Repeating the same argument for the modules $\bigcap_{j=k}^{\nu} Q_{j}(2 \leqq k \leqq \nu)$ successively, we obtain

$$
P(L / M, x) \equiv \sum_{j=1}^{\nu} P\left(L / Q_{j}, x\right)\left(\bmod 1, x, \cdots, x^{r-1}\right) .
$$

Since $P\left(L / Q_{j}, x\right)$ is of degree $r$ if and only if $\mathfrak{F}_{j}$ is of projective dimension $r$ (Lemma 1 ), it follows that $\mu(M ; L)$ is equal to the sum of $\mu\left(Q_{k} ; L\right)$ where $Q_{k}$ are those submodules the prime ideals associated with which are of projective dimension $r$.

Q.E.D.

In concluding this section, we recall that the exponent of a $\mathfrak{P}$ primary submodule $Q$ of $L$ is, by definition, the smallest integer $\sigma$ for which $\mathfrak{P}^{\sigma} L \subset Q$. 


\section{§ 2. Involutive Subspaces}

Let $E$ and $F$ be vector spaces over $K$ of dimensions $m$ and $n$ respectively. Let $F^{*}$ denote the dual space to $F$ and $S^{q}\left(F^{*}\right)$ denote the $q$-th symmetric power on $F^{*}$. The vector space $E \otimes S^{l}\left(F^{*}\right)$ can be considered as the subspace of the space $\left\{E \otimes S^{l-1}\left(F^{*}\right)\right\} \otimes F^{*}=\operatorname{Hom}\left(F, E \otimes S^{l-1}\left(F^{*}\right)\right)$ $(l \geqq 1)$. Let $A$ be a subspace of $E \otimes S^{l}\left(F^{*}\right)$. The prolongation $p A$ of $A$ is defined by

$$
p A=A \otimes F^{*} \cap E \otimes S^{l+1}\left(F^{*}\right) .
$$

We shall denote by $\mathscr{L}_{k}(F)$ the algebraic variety of all $k$-dimensional subspaces of $F$. For an element $F_{k} \in \mathcal{L}_{k}(F), A\left(F_{k}\right)$ is defined to be the space of those elements in $A$ which annihilate the subspace $F_{k}$. Let us put

$$
g_{k}[A]=\min \left\{\operatorname{dim} A\left(F_{k}\right) ; F_{k} \in \mathcal{L}_{k}(F)\right\} \quad(k=0,1, \cdots, n) .
$$

It is obvious that $g_{0}[A] \geqq g_{1}[A] \geqq \cdots \geqq g_{n}[A]=0$. The space $A$ is called an involutive subspace of $\left\{E \otimes S^{l-1}\left(F^{*}\right)\right\} \otimes F^{*}$ if and only if $\operatorname{dim} p A$ is equal to $\sum_{k=0}^{n} g_{k}[A]$ (cf. Kuranishi [12], §6). Throughout this and the next sections, by involutive subspaces we mean involutive subspaces of $\{E$ $\left.E \otimes S^{l-1}\left(F^{*}\right)\right\} \otimes F^{*}$ except when otherwise is expressly stated.

Let $R$ be the graded ring $\sum_{q=0}^{\infty} S^{q}(F)$ and $R_{q}$ be $S^{q}(F)$ ( $S^{0}(F)$ means $K)$. The graded ring $R$ is (isomorphic to) the polynomial ring over $K$ in $n$ variables. We shall denote by $L$ the graded $R$-module $E^{*} \otimes R$ :

$$
L=\sum_{q=0}^{\infty} E^{*} \otimes S^{q}(F), L_{q}=E^{*} \otimes S^{q}(F) .
$$

This module $L$ is finitely generated as an $R$-module, and hence it is a Noetherian $R$-module.

Let $D$ be the annihilator of $A$ in the space $L_{l}$ dual to $E \otimes S^{l}\left(F^{*}\right)$. We shall use the following notation: Let $\Xi$ and $Z$ be subsets of $R$ and $L$ respectively. The set of all finite sums $\sum_{i} \xi_{i} z_{i}$ where $\xi_{i} \in \Xi, z_{i} \in Z$ is denoted by $\Xi L$. Now it is not hard to see that the annihilator of $A\left(F_{k}\right)$ is $D+\left(F_{k} L\right) \cap L_{l}$. Therefore we have

$$
\begin{aligned}
\operatorname{dim} A\left(F_{k}\right) & =\operatorname{dim} L_{l} /\left\{D+\left(F_{k} L\right) \cap L_{l}\right\} \\
& =\operatorname{dim}\left\{L_{l} /\left(F_{k} L\right) \cap L_{l}\right\} /\left\{\left(D+\left(F_{k} L\right) \cap L_{l}\right) /\left(F_{k} L\right) \cap L_{l}\right\}
\end{aligned}
$$




$$
=\left(\begin{array}{c}
l+n-k-1 \\
l
\end{array}\right)-\operatorname{dim} D / D \cap F_{k} L
$$

where $\left(\begin{array}{c}p+q-1 \\ p\end{array}\right)$ is the binomial coefficient $(p+q-1) ! / p ! q !$, which indicates the number of forms of degree $p$ in $q$ variables. Since

$$
\left(\begin{array}{l}
l+n \\
l+1
\end{array}\right)=\left(\begin{array}{c}
l+n-1 \\
l
\end{array}\right)+\left(\begin{array}{c}
l+n-2 \\
l
\end{array}\right)+\cdots+\left(\begin{array}{l}
l \\
l
\end{array}\right),
$$

it follows from the above equalities that

$$
\sum_{k=0}^{n} g_{k}[A]=\left(\begin{array}{l}
l+n \\
l+1
\end{array}\right)-\sum_{k=0}^{n} \max \left\{\operatorname{dim} D / D \cap F_{k} L ; F_{k} \in \mathcal{L}_{k}(F)\right\} .
$$

On the other hand, the annihilator of $p A$ in $L_{l+1}$ coincides with $F D$ (see Kuranishi [12], $\S 6$, Lemma 6.1), and $\operatorname{dim} L_{l+1}=\left(\begin{array}{l}l+n \\ l+1\end{array}\right)$, and hence we have

$$
\operatorname{dim} p A=\left(\begin{array}{l}
l+n \\
l+1
\end{array}\right)-\operatorname{dim} F D
$$

Let $N$ be the homogeneous submodule of $L$ generated by $D ; N=\sum_{q=0}^{\infty} N_{q}$. Clearly $N_{q}=0$ for $q=0,1, \cdots, l-1, N_{l}=D$ and $N_{l+1}=F D$. Then it follows from the above argument that $A$ is an involutive subspace if and only if

$$
\operatorname{dim} N_{l+1}=\sum_{k=0}^{n} \max \left\{\operatorname{dim} N_{l} / N_{l} \cap F_{k} L ; F_{k} \in \mathcal{G}_{k}(F)\right\}
$$

Let $N$ be an arbitrary homogeneous submodule of $L$ such that it is generated by a subspace of $L_{l}$. It is clear that $\operatorname{dim} N_{l} / N_{l} \cap F_{k} L$ where $F_{k} \in \mathcal{L}_{k}(F)$ takes its maximum value outside a certain proper algebraic subvariety of $\mathcal{L}_{k}(F)$. Hence we find no difficulty in proving that there is a sequence $F_{0} \subset F_{1} \subset \cdots \subset F_{n}=F$ where $F_{k} \in \mathscr{G}_{k}(F)$ such that $\operatorname{dim} N_{l} / N_{l} \cap$ $F_{k} L$ attains the maximum value $(k=0,1, \cdots, n)$. The submodule $F_{k} L$ of $L$ is obviously a $\left(F_{k}\right)$-primary submodule of $L,\left(F_{k}\right)$ being the ideal in $R$ generated by $F_{k}$. Accordingly $F_{k+1} z \subset F_{k} L$ implies $z \in F_{k} L$, and hence we have

$$
\left\{z \in N_{l} ; F_{k+1} z \subset F_{k} N_{l}\right\} \subset N_{l} \cap F_{k} L .
$$

The vector space $F_{k+1} N_{l} / F_{k} N_{l}$ is isomorphic to $\xi_{k+1} N_{l} / \hat{\xi}_{k+1} N_{l} \cap F_{k} N_{l}$, 
where $\xi_{k+1}$ is an element of $F_{k+1}$ not belonging to $F_{k}$, and the latter space has the same dimension as the space $N_{l} /\left\{z \in N_{l} ; \xi_{l+1} z \in F_{k} N_{l}\right\}$. Hence we obtain the inequalities

$$
\operatorname{dim} F_{k+1} N_{l} / F_{k} N_{l} \geqq \operatorname{dim} N_{l} / N_{l} \cap F_{k} L \quad(k=0,1, \cdots, n-1) .
$$

Since $N_{l, 1}=F N_{l}$, it follows that

$$
\begin{aligned}
\operatorname{dim} N_{l+1} & =\sum_{k=0}^{n-1} \operatorname{dim} F_{k+1} N_{l} / F_{k} N_{l} \\
& \geqq \sum_{k=0}^{n} \operatorname{dim} N_{l} / N_{l} \cap F_{k} L \\
& =\sum_{k=0}^{n} \max \left\{\operatorname{dim} N_{l} / N_{l} \cap F_{k}{ }^{\prime} L ; F_{k}{ }^{\prime} \in \mathcal{G}_{k}(F)\right\} .
\end{aligned}
$$

Accordingly we have the following

Proposition 1. Let $N$ be a homogeneous submodule of $L$ generated by a subspace of $L_{l} ; N=\sum_{q=i}^{\infty} N_{q}$. Then

$$
\operatorname{dim} N_{l \dashv 1} \geqq \sum_{k=0}^{n} \max \left\{\operatorname{dim} N_{l} / N_{l} \cap F_{k} L ; F_{k} \in \mathcal{G}_{k}(F)\right\}
$$

The equality holds if and only if the subspace of $E \otimes S^{l}\left(F^{*}\right)$ of which annihilator in $L_{l}$ is the space $N_{l}$ is an involutive subspace.

Suppose that $A$ is an involutive subspace. Let $N$ be the submodule of $L$ generated by the annihilator $D$ of $A$. Then equality (4) holds. Let $F_{0} \subset F_{1} \subset \cdots \subset F_{n}$ be a sequence of subspaces of $F$ such that the function $\operatorname{dim} N_{l} / N_{l} \cap F_{k}{ }^{\prime} L$ on $\mathcal{G}_{k}(F)$ attains the maximum value at $F_{k}(0 \leqq k$ $\leqq n)$. Such a sequence $F_{0} \subset F_{1} \subset \cdots \subset F_{n}$ actually exists as we already explained. Combining (6) with (5), we see that the equalities in (5) hold. The argument above (5) indicates that the equalities in (5) hold if and only if

$$
\left\{z \in N_{l} ; F_{k+1} z \subset F_{l} N_{l}\right\}=N_{l} \cap F_{k} L \quad(k=0,1, \cdots, n-1) .
$$

Conversely, we readily see that if these conditions are satisfied for some sequence $F_{0} \subset F_{1} \subset \cdots \subset F_{n}$, then equality (4) holds, that is, $A$ is an involutive subspace. These results can be summarized as follows. 
Lemma 4. Let $A$ and $N$ be as above. $A$ is an involutive subspace if and only if there exists a sequence $F_{0} \subset F_{1} \subset \cdots \subset F_{n}$ where $F_{k} \in \mathcal{L}_{k}(F)$ such that

$\operatorname{dim} F_{k+1} N_{l} / F_{k} N_{l}=\operatorname{dim} N_{l} / N_{l} \cap F_{k} L \quad(k=0,1, \cdots, n-1)$, or such that

$$
\left\{z \in N_{l} ; F_{k+1} z \subset F_{k} N_{l}\right\}=N_{l} \cap F_{k} L \quad(k=0,1, \cdots, n-1) .
$$

In this case the latter conditions are satisfied for $F_{0} \subset F_{1} \subset \cdots \subset F_{n}$ if and only if the function $\operatorname{dim} N_{l} / N_{l} \cap F_{k}{ }^{\prime} L$ on $\mathcal{G}_{k}(F)$ attains the maximum value at $F_{k}(0 \leqq k \leqq n)$.

Using this lemma, we shall deduce the following

Proposition 2. Let $N$ be as in Proposition 1. If equality (4) holds, then the following equality holds for any integer $q \geqq l$ :

$$
\operatorname{dim} N_{q+1}=\sum_{k=0}^{n} \max \left\{\operatorname{dim} N_{q} / N_{q} \cap F_{k} L ; F_{k} \in \mathcal{L}_{k}(F)\right\} .
$$

Proof. By Lemma 4, the desired equality holds if and only if there is a sequence $F_{0} \subset F_{1} \subset \cdots \subset F_{n}$ where $F_{k} \in \mathcal{L}_{k}(F)$ such that

$$
\left\{z \in N_{q} ; F_{k+1} z \subset F_{k} N_{q}\right\}=N_{q} \cap F_{k} L \quad(k=0,1, \cdots, n-1) .
$$

The assumption means that $(7)_{l}$ holds for some sequence $F_{0} \subset F_{1} \subset \cdots \subset F_{n}$ by Lemma 4 . We shall prove that $(7)_{q}$ holds for the same sequence $F_{0} \subset F_{1} \subset \cdots \subset F_{n}$ by induction on $q$. Suppose that $(7)_{q}$ holds. Let us prove $(7)_{q+1}$. To do so, it is sufficient to prove

$$
W=\left\{z \in N_{q+1} ; F_{k+1} z \subset F_{k} N_{q+1}\right\} \supset N_{q+1} \cap F_{k} L
$$

for each fixed $k$ (cf. the argument above (5)). We write $V_{s}=F_{s} N_{q} \cap F_{k} L$. We first prove that $V_{s+1}$ is contained in $V_{k}$ for $s \geqq k$ (, and hence $V_{s+1}=$ $V_{k}$ for $s \geqq k$ ). To show this, it suffices to prove that for each $s \geqq k, V_{s+1}$ is contained in $V_{s}$. Let $z$ be any element of $V_{s+1}$. When $F_{s+1}$ is spanned by $F_{s}$ and $\xi_{s+1}, z$ can be written as follows: $z=\xi_{s^{+1}} z_{s+1}+\hat{z}$ where $z_{s+1} \in N_{q}$, $\hat{z} \in F_{s} N_{q}$. Since $\xi_{s+1} \notin F_{s}, z_{s+1}$ is necessarily an element of $F_{s} L \cap N_{q}=V_{s}$. It follows from the induction assumption (7) $)_{q}$ that $\xi_{s+1} \quad z_{s+1} \in F_{s} N_{q}$. Consequently $z=\xi_{s+1} z_{s+1}+\hat{z}$ belongs to $F_{s} N_{q}$. This implies that $z \in V_{s}$. Hence $V_{s+1} \subset V_{s}$ as desired. We now prove (8). Obviously $V_{k} \subset W$. 
Therefore, by what we have just deduced, $V_{s} \subset W$ for all $s=0,1, \cdots, n$. In particular we have $V_{n} \subset W$. Since $N_{q+1}=F_{n} N_{q}, \quad V_{n}=N_{q+1} \cap F_{k} L$. Combining these, we have (8) as desired.

Q.E.D.

Remark. The above proof also implies the following: If $F_{0} \subset F_{1}$ $\subset \cdots \subset F_{n}$ where $F_{k} \in \mathcal{G}_{k}(F)$ is a sequence such that $(7)_{l}$ holds. Then $F_{s} N_{q} \cap F_{k} L=F_{k} N_{q}$ for $s \geqq k$ and $q \geqq l$.

Note. It is well-known that $\operatorname{dim} p A \leqq \sum_{k=0}^{n} g_{k}[A]$, and that if $A$ is an involutive subspace, then $p A$ is also an involutive subspace of $\{E$ $\left.\otimes S^{l}\left(F^{*}\right)\right\} \otimes F^{*}$ (cf. Kuranishi [12]). Propositions 1 and 2 are equivalent to these facts respectively. The reason why we give our direct proofs is that the discussion in the course of the proofs is needed in the following investigation.

\section{§ 3. Modules Associated with Involutive Subspaces}

Let $R$ and $L$ be the same ring and $R$-module as in Section 2 respectively. Let $N$ be a homogeneous submodule of $L$. We denote by $\mathfrak{x}$ the maximal ideal in $R ; \mathfrak{X}=\sum_{q=1}^{\infty} R_{q}$.

Notation. We denote by $H(N)$ the smallest (homogeneous) submodule $M$ of $L$ which contains $N$ and which possesses the property

$$
\mathfrak{x} z \subset M \text { implies } z \in M \text {. }
$$

The submodule $H(N)$ is constructed from $N$ as follows. Writing $M^{(0)}=N$, we define the submodules $M^{(s)}$ of $L$ inductively by

$$
M^{(s+1)}=\left\{z \in L ; \mathfrak{t} z \subset M^{(s)}\right\} \quad(s=0,1,2, \cdots) .
$$

It is obvious that $M^{(0)} \subset M^{(1)} \subset M^{(2)} \subset \cdots$. Since $L$ is Noetherian, there exists an integer $s$ such that $M^{(s)}=M^{(s+1)}=\cdots$. Then $H(N)$ is nothing else than $\bigcup_{i=0}^{\infty} M^{(i)}=M^{(s)}$. It is to be noted that $H(N) \cap L_{q}$ coincides with $N \cap L_{q}$ for all sufficiently large $q$. Conversely, if $N$ and $N^{\prime}$ be two homogeneous submodules of $L$ such that $N \cap L_{q}=N^{\prime} \cap L_{q}$ for all large $q$, then $H(N)=H\left(N^{\prime}\right)$.

We can interpret the process of constructing $H(N)$ from $N$ quite differently, applying the theory of primary decompositions: A reduced 
primary decomposition of $H(N)$ in $L$ is obtained from that of $N$ by omitting the component which is $\mathfrak{t}$-primary (if it exists). When $N$ is itself $\mathfrak{X}$-primary, by this statement we mean that $H(N)=L$. Let us prove this fact. Let $Q$ be a $\mathfrak{B}$-primary submodule of $L$. We first prove that if $\mathfrak{P} \varsubsetneqq \mathbb{X}$, then $H(Q)=Q$. It is enough to show that $Q$ has the

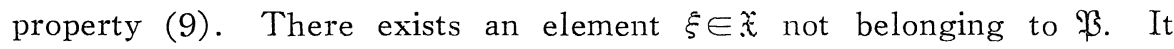
follows from the very definition of primary modules that $\xi z \in Q$ implies $z \in Q$. This shows that $\mathfrak{X} z \subset Q$ implies $z \in Q$. Hence our desired result follows. We next prove that if $\mathfrak{P}=\mathfrak{X}$, then $H(Q)=L$. Since $\mathfrak{X}^{\sigma} L \subset Q$ for some positive integer $\sigma$ and $L$ is generated by $L_{0}, Q \cap L_{q}=L_{q}$ for $q \geqq \sigma$. From this and the definition of $H(Q)$ it follows that $H(Q)=L$. We now prove our original assertion. Let $N=\bigcap_{j=1}^{p} N_{(j)}$ be a reduced primary decomposition in $L$. It is not hard to show that $H(N)=\bigcap_{j=1}^{p}$ $H\left(N_{(j)}\right)$ (cf. the first process of constructing $H(N)$ from $N$ ). Hence our assertion follows from what we have just deduced.

Let $A$ be a subspace of $E \otimes S^{l}\left(F^{*}\right)$. We associate with $A$ an $R$ submodule of $L$ as follows.

Definition. Let $N$ be the submodule of $L$ generated by the annihilator $D$ of $A$ in $L_{l}$. We define the (homogeneous) submodule $M(A)$ of $L$ to be $H(N)$, and call it the module associated with $A$.

It is clear that the module associated with $p A$ coincides with $M(A)$; In other words, the module associated with a space is unchanged under the prolongation. Moreover there never exists the maximal ideal $\mathfrak{x}$ in $\operatorname{Ass}(L / M(A))$ as we explained above. These are the reasons why we associate with $A$ the module $M(A)=H(N)$ instead of $N$ itself.

In the remaining part of this section, we devote ourselves to investigating the structure of the module $M(A)$ under the condition that $A$ is an involutive subspace. A link between $M(A)$ and $N$ (see the above definition) is given by the following lemma. We write: $M(A)=$ $\sum_{q=0}^{\infty} M_{q}(A), N=\sum_{q=0}^{\infty} N_{q}$ (direct sum decomposition of graded modules). Remark that $N_{q}=0$ for $q=0,1, \cdots, l-1$ and $N_{l}=D$. 
Lemma 5. Suppose that $A$ is an involutive subspace. Then. under the above circumstances, $M_{q}(A)=N_{q}$ for all $q \geqq l$.

This lemma indicates that the elements to be added to $N$ in order to obtain the module $M(A)$ (if they exist) are of degrees less than $l$. Of course, this is not valid in general when $A$ is not an involutive subspace.

Proof. For brevity, we write $M=M(A), M_{q}=M_{q}(A)$. It follows from the definition of $H(N)$ that there exists an integer $s$ such that $M_{q}=N_{q}$ for $q \geqq s$. Assume $s>l$. Let us prove $M_{s-1}=N_{s-1}$. Obviously $M_{s-1} \supset N_{s-1}$. Let $F_{k}$ be an element of $\mathcal{G}_{k}(F)(0 \leqq k \leqq \mathrm{n})$. The natural homomorphism from $N_{s-1} / N_{s-1} \cap F_{k} L$ to $M_{s-1} / \Lambda_{s-1} \cap F_{k} L$ is obviously injective. Therefore we have

$$
\operatorname{dim} N_{s-1} / N_{s-1} \cap F_{k} L \leqq \operatorname{dim} M_{s-1} / M_{s-1} \cap F_{k} L \quad(0 \leqq k \leqq n)
$$

It follows from this that

$$
\begin{aligned}
\sum_{k=0}^{n} \max & \left\{\operatorname{dim} N_{s-1} / N_{s-1} \cap F_{k} L ; F_{k} \in \mathscr{L}_{k}(F)\right\} \\
\leqq & \leqq \sum_{k=0}^{n} \max \left\{\operatorname{dim} M_{s-1} / M_{s-1} \cap F_{k} L ; F_{k} \in \mathscr{L}_{k}(F)\right\} .
\end{aligned}
$$

By virtue of Propositions 1 and 2 in $\S 2$, the number on the left side is equal to $\operatorname{dim} N_{s}$. Besides, applying Proposition 1 to the module generated by $M_{s-1}$, we know that the number on the right side is equal to or less than $\operatorname{dim} M_{s}$. Combining these facts with $\operatorname{dim} M_{s}=\operatorname{dim} N_{s}$, we conclude that the above inequality is actually the equality. Since each term on the left side is equal to or less than the corresponding term on the right side, as a consequence of the equality, we have $\operatorname{dim} M_{s-1}$ $=\operatorname{dim} N_{s-1}$. Hence $M_{s-1}=N_{s-1}$. If $s-1>l$, by the same reasoning, we have $M_{s-2}=N_{s-2}$. Proceed step by step, we can complete the proof of the fact that $M_{q}=N_{a}$ for all $q \geqq l$.

Q.E.D.

Let us calculate the degree of the characteristic polynomial of $L / M(A)$ and $" \iota(M(A) ; L)$ for an involutive subspace $A$. 
Lemma 6. Suppose that $A$ is an involutive subspace and that $g_{p-1}[A]>0, g_{p}[A]=0$. Then the greatest projective dimension of the prime ideals in $\operatorname{Ass}(L / M(A))$ is equal to $p-1$. Furthermore $\mu(M(A) ; L)$ is equal to $g_{p-1}[A]$.

Proof. We write: $M=M(A)$. The annihilator of $A\left(F_{p}\right)$ in $L_{l}$ is $M_{l}+\left(F_{p} L\right) \cap L_{l}$ (cf. Lemma 5), and hence the assumption $g_{p}[A]=0$ implies that $\left(M+F_{p} L\right) \cap L_{l}=L_{l}$ for any $F_{p} \in \mathcal{L}_{p}(F)$ outside a proper subvariety. Therefore we can prove without any difficulty that there is a sequence $F_{0} \subset F_{1} \subset \cdots \subset F_{n}$ where $F_{k} \in \mathscr{L}_{k}(F)$ for which the conditions in Lemma 4 in $\S 2$ are satisfied and moreover $\left(M+F_{p} L\right) \cap L_{l}=L_{l}$. Then by Remark at the end of $\S 2$ and by Lemma 5 , we have

$$
F_{p-1} L \cap F_{k} M_{l}=F_{p-1} M_{l} \text { for } k \geqq p-1 \text {. }
$$

Let us prove the equality

$$
\text { (11) } \operatorname{dim} M_{l \rightarrow 1} / M_{l+1} \cap F_{p-1} L=\left(\begin{array}{c}
l+n-p \\
l+1
\end{array}\right)+\operatorname{dim} M_{l} / M_{l} \cap F_{p-1} L .
$$

Remarking Lemma 5, we have the equalities

$$
\begin{aligned}
\operatorname{dim} & M_{l+1} / M_{l+1} \cap F_{p-1} L \\
& =\sum_{k=p}^{n} \operatorname{dim} F_{k} M_{l} /\left\{F_{p-1} L \cap F_{k} M_{l}+F_{k-1} M_{l}\right\} \\
& =\sum_{k=p}^{n} \operatorname{dim} F_{k} M_{l} / F_{k-1} M_{l}(\text { by }(10)) \\
& \left.=\sum_{k=p}^{n} \operatorname{dim} M_{l} / M_{l} \cap F_{k-1} L \text { (by the choice of } F_{k}\right) .
\end{aligned}
$$

Obviously $\left(M+F_{k} L\right) \cap L_{l}=L_{l}$ for $k \geqq p$, and hence

$$
\operatorname{dim} M_{l} / M_{l} \cap F_{k} L=\operatorname{dim} L_{l} /\left(F_{k} L\right) \cap L_{l}=\left(\begin{array}{c}
l+n-k-1 \\
l
\end{array}\right) .
$$

Since $\sum_{k=p}^{n-1}\left(\begin{array}{c}l+n-k-1 \\ l\end{array}\right)=\left(\begin{array}{c}l+n-p \\ l+1\end{array}\right)$, it follows that equality (11) is indeed valid.

Let us put

$$
\begin{aligned}
\delta_{q}\left(F_{p-1}\right) & =\operatorname{dim} L_{q} /\left(M+F_{p-1} L\right) \cap L_{q} \\
& =\left(\begin{array}{c}
q+n-p \\
q
\end{array}\right)-\operatorname{dim} M_{q} / M_{q} \cap F_{p-1} L,
\end{aligned}
$$




$$
\delta_{q}=\min \left\{\delta_{q}\left(F_{p-1}\right) ; F_{p-1} \in \mathcal{L}_{p-1}(F)\right\} .
$$

Equality (11) means that $\delta_{l}\left(F_{p-1}\right)=\delta_{l+1}\left(F_{p-1}\right)$. Since the condition in Lemma 4 is satisfied by our assumption,

$$
\delta_{l}=\delta_{l}\left(F_{p-1}\right)=\delta_{l+1}\left(F_{p-1}\right)=\delta_{l+1} .
$$

Since $p A$ is an involutive subspace of $\left\{E \otimes S^{l}\left(F^{*}\right)\right\} \otimes F^{*}$, (cf. Note at the end of $\S 2$ ), we can prove in just the same manner that $\delta_{l+1}=\delta_{l+2}$; and so on. Thus we have $\delta_{l}=\delta_{l_{11}}=\delta_{1+2}=\cdots$.

Now the annihilator of $A\left(F_{p-1}\right)$ in $L_{l}$ is $D+\left(F_{p-1} L\right) \cap L_{l}=(M$ $\left.+F_{p-1} L\right) \cap L_{l}$, and hence $g_{p-1}[A]=\delta_{l}>0$. The fact that $\delta_{q}$ is positive means that $\left(M+F_{p-1} L\right) \cap L_{q} \neq L_{q}$ for any $F_{p-1} \in \mathcal{G}_{p-1}(F)$. Hence what we have shown above implies that $p$ is equal to the smallest integer $k$ for which there is an element $F_{k} \in \mathcal{L}_{k}(F)$ such that $\left(M+F_{k} L\right) \cap L_{q}=L_{q}$. Applying (i) in Lemma 2 in $\S 1$, we conclude that the degree of the characteristic module $P(L / M(A), x)$ is equal to $p-1$. This means that the greatest projective dimension of the prime ideals in Ass $(L / M(A))$ is equal to $p-1$ by Lemma 1 in $\S 1$. Moreover applying (ii) of Lemma 2, we have $\mu(M(A) ; L)=\delta_{q}$ for large $q$. Combining this with the fact $g_{p-1}[A]=\delta_{l}=\delta_{l+1}=\cdots$, we obtain $g_{p-1}[A]=\mu(M(A) ; L)$ as desired.

Q.E.D.

We note that under the circumstances in Lemma 6 , the integer $g_{p}[A]$ is unchanged under the prolongation; $g_{p}[A]=g_{p}[p A]$. This is an immediate consequence of Lemma 6 , for $M(A)$ is also the module associated with $p A$.

Applying Theorem $I$ in the preceding section and using Lemma 6, we have the following fundamental result.

Theorem II. Suppose that $A$ is an involutive subspace of $\{E$ $\left.\otimes S^{l-1}\left(F^{*}\right)\right\} \otimes F^{*}$ and that $g_{p-1}[A]>0, g_{p}[A]=0$. Let $M(A)=\bigcap_{j=1}^{\nu} Q_{j}$ be a reduced primary decomposition of the module $M(A)$ associated with $A$ in $L$, and let $Q_{j}$ be $\mathfrak{P}_{j}$-primary in $L$.

Then the greatest projective dimension of the prime ideals in Ass $(L / M(A))=\left\{\mathfrak{P}_{1}, \cdots, \mathfrak{P}_{\nu}\right\}$ is equal to $p-1$, and the following is valid:

$$
g_{p-1}[A]=\sum_{\text {proj dim } \mathfrak{P}_{j=p-1}} \mu\left(Q_{j}: L\right) .
$$




\section{Part II. Involutive Systems of Partial Differential Equations}

\section{$\S 4 . \quad$ Criterion of Involution}

Let $(\mathcal{M}, \mathcal{N}, \rho)$ be an analytic fibered manifold where $\rho$ is the projection from $\mathscr{M}$ onto $\mathcal{N}$, and $J^{l}(\mathcal{M}, \mathcal{N}, \rho)$ be the space of $l$-jets of crosssections of $(\mathscr{M}, \Re, \rho)$. We shall often denote the space $J^{l}(\mathscr{M}, \Re, \rho)$ briefly by $J^{l}$. The sheaf of germs of analytic functions on an open set $U$ in $J^{l}$ is denoted by $\mathcal{O}(U)$. A locally finitely generated subsheaf of ideals of the sheaf of rings $\mathcal{O}(U)$ is called a system of partial differential equations of order $l$ in $(\mathscr{M}, \mathcal{N}, \rho)$. A point $X$ of $J^{l}$ is called an integral one of $\Phi$ if every $\varphi \in \Phi_{X}$ vanishes at $X$, where $\Phi_{X}$ denotes the ring of germs of sections of $\Phi$ at $X$. The set of integral points of $\Phi$ is denoted by $I \Phi$. An integral point $X$ is called an ordinary one if $\Phi=0$ gives a regular local equation of the variety $I \Phi$ around $X$. A cross-section $f$ of $(\mathcal{M}, \mathcal{\Omega}, \rho)$ over an open set $V$ in $\Omega$ is said to be a solution of $\Phi$ if and only if the $l$-jet $j_{a}{ }^{l}(f)$ of $f$ at $a$ is an integral point of $\Phi$ for every $a$ in $V$.

Let $\rho_{k}{ }^{l}$ be the projection from $J^{l}$ onto $J^{k}$ defined by $\rho_{k}{ }^{l}\left(j_{a}{ }^{l}(f)\right)=j_{a}{ }^{k}$ ( $f)(l \geqq k \geqq 0)$ and $\rho_{-1}^{l}$ be $\rho \circ \rho_{0}^{l}$. For each point $X \in J^{l}$ and $\bar{X}=\rho_{l-1}^{l} X$, one has the injection $i$ from $\mathcal{O}_{\bar{X}}\left(J^{l-1}\right)$ into $\mathcal{O}_{\bar{X}}\left(J^{l}\right)$ defined by $i \varphi=\varphi \circ \rho_{l-1}^{l}$. We identify $\mathcal{O}_{\bar{X}}\left(J^{l-1}\right)$ with its image $i \Theta_{\bar{X}}\left(J^{l-1}\right)$.

Let $\left(\rho_{l-1}^{l}\right)_{*}$ be the differential of the mapping $\rho_{l-1}^{l}$ at $X ;\left(\rho_{l-1}^{l}\right)_{*}$ : $T_{X}\left(J^{l}\right) \rightarrow T_{\bar{X}}\left(J^{l-1}\right)$, where $T_{X}\left(J^{l}\right)$ denotes the tangent space to $J^{l}$ at $X$. The kernel of $\left(\rho_{l-1}^{l}\right)_{*}$ is denoted by $Q_{X}\left(J^{l}\right)$. For brevity, we shall write $a=\rho_{-1}^{l} X$ and $b=\rho_{0}^{l} X$.

Let $Q_{b}(\mathscr{M})$ be the kernel of the mapping $\rho_{*}: T_{b}(\mathscr{M}) \rightarrow T_{a}(\mathscr{N})$. The space $Q_{X}\left(J^{l}\right)$ can be identified with $Q_{b}(\mathscr{M}) \otimes S^{l}\left(T_{a}{ }^{*}(\mathcal{R})\right)$. The subspace $C_{X}(\Phi)$ of $Q_{X}\left(J^{l}\right)$ is defined by

$$
C_{X}(\Phi)=\left\{\mathscr{X} \in Q_{X}\left(J^{l}\right) ; \mathscr{X}(\varphi)=0 \text { for every } \varphi \in \Phi_{X}\right\} \quad(X \in I \Phi) .
$$

This space is considered canonically as a subspace of $Q_{\bar{X}}\left(J^{l-1}\right) \otimes T_{a}{ }^{*}$ $(\Re)$. The notion of involutive subspaces of $Q_{\bar{X}}\left(J^{l-1}\right) \otimes T_{a}{ }^{*}(\mathcal{R})$ can be introduced as we recalled in $\S 2$ (See Kuranishi [12], §6). The prolongation of such subspaces is also defined (see $\S 2$ ). In particular, $p C_{X}(\Phi)$ is defined by 


$$
p C_{X}(\Phi)=C_{X}(\Phi) \otimes T_{a} *(\Re) \cap Q_{\bar{X}}\left(J^{l-1}\right) \otimes S^{2}\left(T_{a}^{*}(\mathcal{I})\right) .
$$

The (total) prolongation of $\Phi$ is defined as follows. Let $\varphi$ be a function on an open set $U$ in $J^{l}$ and $\xi$ be a vector field on $\rho_{-1}^{l} U \subset \eta$. Let $\varphi_{\xi}$ denote a function on $\left(\rho_{l}^{l+1}\right)^{-1} \chi l$ defined by

$$
\varphi_{\xi}\left(j_{a}{ }^{l+1}(f)\right)=\xi\left(\varphi\left(j_{a}^{l}(f)\right) .\right.
$$

For each open set $\widetilde{\widetilde{U}}$ in $J^{l 11}$, we denote by $\mathcal{L}_{\widetilde{u}}$ that ideal in the ring of analytic functions on $\widetilde{\widetilde{U}}$ which is generated by all sections of the sheaf $\Phi$ over $\rho_{l}{ }^{l+1} \widetilde{U}$ and all $\varphi_{\xi}$ constructed from every section $\varphi$ of $\Phi$ over $\rho_{l}{ }^{l\urcorner 1} \widetilde{\mathcal{U}}$ and every vector field $\xi$ on $\rho_{-1}^{l+1} \widetilde{\mathcal{U}}$. Let $\rho_{\widetilde{q} \widetilde{\sim}}$ denote the restriction mapping from $\mathscr{L}_{\widetilde{q}}$ to $\mathcal{L}_{\widetilde{U}}$, where $\widetilde{U} \subset \widetilde{\mathcal{V}}$. Then we have the presheaf $\left\{\mathcal{L}_{\widetilde{u}}, \rho_{\widetilde{u} \widetilde{v}}\right\}$ over $J^{l+1}$. The prolongation $\rho \Phi$ of $\Phi$ is defined to be the sheaf over $J^{l+1}$ associated with the presheaf $\left\{\mathcal{L}_{\widetilde{u}}, \rho_{\tilde{u} \widetilde{\mho}}\right\}$.

Let $\widetilde{X}$ be a point of $\left(\rho_{l}{ }^{l+1}\right)^{-1} X . \quad(p \Phi) \widetilde{X} \cap \mathcal{O}_{X}\left(J^{l}\right)$ is independent of the choice of such $\widetilde{X}$. $\Phi$ is said to be p-closed at $X$ if and only if $(p \Phi)_{\widetilde{X}} \cap \Theta_{X}\left(J^{l}\right)$ is contained in $\Phi_{X}$; When $X$ is an ordinary integral point, $\Phi$ is $p$-closed at $X$ if and only if there exists a neighbourhood $U$ of $X$ such that the mapping $\rho_{l}{ }^{l+1}$ from $I(p \Phi) \cap\left(\rho_{l}{ }^{l+1}\right)^{-1} U$ to $I \Phi \cap U$ is surjective (Matsuda [13]).

M. Kuranishi [12] gave a modern characterizalion of involutive systems of partial differential equations. Let us recall the criterion of involution given by M. Matsuda [13].

Theorem A. $\Phi$ is involutive at $X_{0}$ if and only if the following four conditions are satisfied:

(i) $X_{0}$ is an ordinary integral point of $\Phi$.

(ii) $\operatorname{dim} p C_{X}(\Phi)$ remains constant on a neighbourhood of $X_{0}$ in $I \Phi$.

(iii) $C_{Y_{0}}(\Phi)$ is an involutive subspace of $Q_{\bar{X}_{0}}\left(J^{l-1}\right) \otimes T_{a_{0}}^{*}(\Re)\left(\bar{X}_{0}\right.$ $\left.=\rho_{l-1}^{l} X_{0}, a_{0}=\rho_{-1}^{l} X_{0}\right)$.

(iv) $\Phi$ is p-closed at $X_{0}$.

Note. If $\Phi$ is involutive at $X_{0}$, then $\Phi$ is involutive at each point on a neighbourhood of $X_{0}$ in $I \Phi$.

Let $\left(x_{1}, \cdots, x_{n}, y_{1}, \cdots, y_{m}\right)$ be a coordinate system of $\mathcal{M}$ on $U$ ( $n$ 
$=\operatorname{dim} \Re, n+m=\operatorname{dim} \mathscr{M})$ such that there exists a coordinate system $\left(x_{1}{ }^{\prime}\right.$, $\left.\cdots, x_{n}{ }^{\prime}\right)$ of $\eta$ on $\rho U$ satisfying $x_{i}=x_{i}{ }^{\prime} \cdot \rho(1 \leqq i \leqq n)$. Then a coordinate system of $J^{l}$ on $\left(\rho_{0}^{l}\right)^{-1} U$ is given by

$$
\left(x_{i}, y_{\alpha}, p_{\alpha}^{i}, p_{\alpha}^{i_{1} i_{2}}, \cdots, p_{\alpha}^{i_{1} \cdots i_{2}} ; 1 \leqq \alpha \leqq m, 1 \leqq i, i_{k} \leqq n\right),
$$

where

$$
p_{\alpha}{ }^{i_{1} \cdots i_{k}}\left(j_{a}{ }^{l}(f)\right)=\frac{\partial^{k} f_{\alpha}}{\partial x_{i_{1}} \cdots \partial x_{i_{k}}}(a), f_{\alpha} \text { being } y_{\alpha} \text {-coordinate of } f
$$

By the contact forms of orders up to and including $l$, we mean the following Pfaffian forms on $J^{l}(\mathcal{M}, \mathcal{N}, \rho)$ :

$$
\begin{cases}d y_{\alpha}-\sum_{i=1}^{n} p_{\alpha}{ }^{i} d x_{i} & (1 \leqq \alpha \leqq m), \\
d p_{\alpha}{ }^{i_{1} \cdots i_{k}}-\sum_{i=1}^{n} p_{\alpha}{ }^{i_{1} \cdots i_{k} i} d x_{i} & \left(\begin{array}{l}
1 \leqq \alpha \leqq m, 1 \leqq k<l \\
1 \leqq i_{1}, \cdots, i_{k} \leqq n
\end{array}\right) .\end{cases}
$$

In the usual way, one can associate with $\Phi$ the exterior differential system $\Sigma(\Phi)$ defined on the domain of $\Phi$ in $J^{l}$ which is generated as a differential ideal by $\Phi$ and the contact forms of orders up to and including $l$. Every solution of $\Phi$ corresponds in a one-to-one manner to an $n$-dimensional integral manifold $\mathfrak{M}$ of $\Sigma(\Phi)$ such that $\operatorname{dim}\left(\rho_{-1}^{l}\right)_{*} T(\mathcal{M})=n$.

The differential system $\Sigma(\Phi)$ is said to be involutive at $X$ with respect to $\Omega$ if and only if the following two conditions (i) and (ii) are satisfied:

(i) There exists at least one $n$-dimensional integral element $E_{n}$ of $\Sigma(\Phi)$ and of origin $X$ such that $\operatorname{dim}\left(\rho_{-1}^{l}\right)_{*} E_{n}=n$,

(ii) Every such integral element $E_{n}$ is an ordinary one in the sense that there exists a chain of integral elements $E_{0} \subset E_{1} \subset \cdots \subset E_{n}$ where each $E_{k}$ except the last $E_{n}$ is a regular integral element of $\Sigma(\Phi)$ and of dimension $k$ (such a chain is called a regular chain.). (cf. Cartan [4], Chap. V; Kuranishi [11], Definition I. 17; Kähler [8].)

The link between two concepts of involution is clarified by the following theorem (Kuranishi [12], Appendix; Matsuda [15]).

Theorem B. $\Phi$ is involutive at $X$ if and only if $\Sigma(\Phi)$ is involutive at $X$ with respect to $\mathcal{\Omega}$. 
We finally recall the following theorem due to E. Cartan and Y. Matsushima [16].

Theorem $\mathbb{C}$. If $\Phi$ is involutive at $X$, then $p \Phi$ is involutive at each point on $I(p \Phi) \cap\left(\rho_{l}{ }^{l+1}\right)^{-1} X$.

\section{§5. The Characteristic Module}

Let us denote by $K$ the field of real or complex numbers according as our discussion is carried out in the real or complex analytic category. Let $X$ be an integral point of $\Phi$. The spaces $Q_{b}(\mathcal{M})$ and $T_{a}(\Re)$ are vector spaces over $K$ of dimensions $m$ and $n$ respectively (cf. $\S 4$ ). We shall apply the theory developed in Part $\mathrm{I}$ in which we shall set $E$ $=Q_{b}(\mathscr{M})$ and $F=T_{a}(\Re)$. We shall also use freely the same notations as in Part I.

Suppose that $\Phi$ is involutive at $X$. We begin with recalling Cartan's characters of $\Phi$ at $X$. From the space $C_{X}(\Phi)$ we obtain the following decreasing sequence of integers (see $\S 2$ ):

$$
g_{0}\left[C_{-Y}(\Phi)\right] \geqq g_{1}\left[C_{X}(\Phi)\right] \geqq \cdots \geqq g_{n}\left[C_{X}(\Phi)\right]=0 .
$$

For brevity we write $g_{k}(X)=g_{k}\left[C_{X}(\Phi)\right]$. One can prove the following inequalities (see Kuranishi [12], § 6):

$$
g_{k-2}(X)-g_{k-1}(X) \geqq g_{k-1}(X)-g_{k}(X) \quad(k=2, \cdots, n) .
$$

Let us put

$$
s_{k}(X)=g_{k-1}(X)-g_{k}(X) \quad(k=1,2, \cdots, n) .
$$

These numbers $s_{k}(X)$ are indeed those characters of the differential system $\Sigma(\Phi)$ associated with $\emptyset$ which were introduced by E. Cartan [2] (also see Kähler [8], p. 55). The above inequalities imply that $s_{1}(X) \geqq s_{2}(X)$ $\geqq \cdots \geqq s_{n}(X) \geqq 0$. It should be noted that the inequalities of this type valid for Cartan's characters are always valid only in case of an exterior differential system generated as a differential ideal by differential forms of degree $\leqq 1$. We call the number $s_{k}(X)$ Cartan's character of order $k$ of $\Phi$ at $X$. The characters $s_{k}(X)$ play an important role in the CartanKähler existence theorem for the differential system $\Sigma(\Phi)$ and they indi- 
cate the numbers of arbitrary functions upon which depends the general integral manifold of dimension $n$ (cf. Kähler [8], p. 55). In case of partial differential equations, however, it is more adequate to introduce the new numbers

$$
\sigma_{k}(X)=s_{k}(X)-s_{k+1}(X)(1 \leqq k<n), \quad \sigma_{n}(X)=s_{n}(X)
$$

as M. Kuranishi did in his lecture note [12]. In fact, it can be seen that the latter numbers $\sigma_{k}(X)$ indicate more precisely the numbers of arbitrary functions upon which depends the general solution of $\Phi$ (Compare the existence proof in Cartan [2] or Kähler [8] with that in Kuranishi [12] or Matsuda [14]). Let $s_{p}(X)$ (resp. $\sigma_{r}(X)$ ) be the last non-vanishing number in $s_{1}(X), \cdots, s_{n}(X)$ (resp. $\sigma_{1}(X), \cdots, \sigma_{n}(X)$ ). Then it is clear that $p=r$ and $s_{p}(X)=\sigma_{r}(X)$. It is this character $s_{p}(X)$ which is of great importance in the following investigation. One reason why the character $s_{p}(X)$ is especially important consists in the fact that it is unchanged under the total prolongation; more precisely, the character of order $p$ of $p \Phi$ at each point of $I(p \Phi) \cap\left(\rho_{l}{ }^{l+1}\right)^{-1} X$ is equal to $s_{p}(X)$ (cf. Lemma 6 in $\S 3$ ).

The characters $s_{k}(X)$ remain constant on a connected open set $C$ in $I \Phi$ at each point of which $\Phi$ is involutive. In particular, they are constant on a neighbourhood in $I \Phi$ of a point at which $\Phi$ is involutive. In fact, $C_{X}(\Phi)$ is an involutive subspace of $Q_{\bar{X}}\left(J^{l-1}\right) \otimes T_{a}{ }^{*}(\Re)$ if and only if $\operatorname{dim} p C_{X}(\Phi)=\sum_{k=0}^{n} g_{k}(X)$ (cf. $\S 2$ ). Hence we conclude from Theorem A that $\operatorname{dim} p C_{X}(\Phi)$ remains constant on $C \mathcal{V}$ and it is equal to $\sum_{k=0}^{n} g_{k}(X)$ for each $X \in C)$. Since the functions $g_{k}(X)$ are upper semi-continuous and integral-valued, it follows that the functions $g_{k}(X)$ are constant on $C$ ). Therefore the characters $s_{k}(X)$ are also so.

We now introduce the important concept "characteristic module". To indicate precisely the dependence on the point $X$, we write:

$$
\begin{aligned}
& R_{q}(X)=S^{q}\left(T_{a}^{*}(\Re)\right), R(X)=\sum_{q=0}^{\infty} R_{q}(X) \\
& L_{q}(X)=Q_{b}(\mathcal{M}) \otimes S^{q}\left(T_{a} *(\Re)\right), L(X)=\sum_{q=0}^{\infty} L_{q}(X) .
\end{aligned}
$$

Definition. We define the characteristic module $M(X)$ of $\Phi$ at $X$ to be the module associated with the space $C_{X}(\Phi)$ in the sense of Section 3. 
The module $M(X)$ is a homogeneous submodule of the $R(X)$-module $L(X)$. It follows immediately from the definition that the characteristic module is unchanged under the prolongation in the sense that the characteristic module of $p \Phi$ at $\widetilde{X}$ coincides with that of $\Phi$ at $X$, where $\widetilde{X}$ is any integral point of $p \Phi$ satisfying $\rho_{l}{ }^{l+1} \widetilde{X}=X$.

Suppose that $s_{p}(X)>0$ and $s_{p+1}(X)=\cdots=s_{n}(X)=0$. Clearly this assumption means that $g_{p-1}(X)>0$ and $g_{p}(X)=0$. Moreover $s_{p}(X)$ is equal to $g_{p-1}(X)$. Hence applying Theorem II in $\S 3$, we have the following fundamental result.

Theorem III. Suppose that $\Phi$ is involutive at $X$ and that $s_{p}(X)$ $>0, s_{p+1}(X)=\cdots=s_{n}(X)=0(1 \leqq p \leqq n)$. Let $M(X)=\bigcap_{j=1}^{\nu} Q_{j}$ be a reduced primary decomposition of the characteristic module $M(X)$ of $\Phi$ at $X$ in $L(X)$ where $Q_{j}$ is a i $_{j}$-primary homogeneous submodule of $L(X) \quad(1 \leqq j \leqq \nu)$.

Then the greatest projective dimension of the homogeneous prime ideals $\mathfrak{P}_{1}, \mathfrak{F}_{2}, \cdots, \mathfrak{P}_{\nu}$ is equal to $p-1$ and the following equality is valid:

$$
s_{p}(X)=\sum_{\text {proj dim } \mathfrak{\beta}_{j=p-1}} \mu\left(Q_{j} ; L(X)\right),
$$

where $\mu\left(Q_{j} ; L(X)\right)$ denotes the multiplicity of $Q_{j}$ with respect to $L(X)$.

Remark. Theorem III is the complete generalization of our previous result ([10], Theorem 2 ).

\section{§ 6. Characteristics}

This section is devoted to the investigation on what is called characteristics in the sense of Monge or Cauchy. It is the primary decomposition of the characteristic module which enables us to clarify the structure of characteristics for general non-linear systems of partial differential equations.

Let $X$ be an integral point of $\Phi$. Obviously it is also an integral point of the differential system $\Sigma(\Phi)$ canonically associated with $\Phi$ (see $\S 4)$. Let $E_{p}$ be a $p$-dimensional integral element of $\Sigma(\Phi)$ and of origin 
$X(0 \leqq p \leqq n)$. The space of such vectors $\Gamma \in T_{X}\left(J^{l}\right)$ that $E_{p}$ and $\Gamma$ spann an integral element of $\Sigma(\Phi)$ is denoted by $H\left(E_{p}\right)$ and it is usually called the polar element. Since $\Sigma(\Phi)$ is generated by differential forms of degree $\leqq 2$ as an exterior algebra, a subspace $E$ of $T_{Y}\left(J^{l}\right)$ is an integral element of $\Sigma(\Phi)$ if and only if any linear element contained in $E$ is an integral one and furthermore, for any pair $\left\{E_{1}, E_{1}{ }^{\prime}\right\}$ of one-dimensional subspaces of $E, E_{1}$ and $E_{1}{ }^{\prime}$ spann an integral element, in other words, $H\left(E_{1}\right)$ contains $E_{1}{ }^{\prime}$. Let $E_{1}$ be a linear element of origin $X$ on which the contact forms of orders up to and including $l$ vanish. $E_{1}$ is spanned by the vector $\Gamma$ expressed in the following manner by using the local coordinate system of $J^{l}$ around $X$ (cf. $\S 4$ ):

$$
\Gamma=\sum_{i=1}^{n} \lambda_{i} \frac{d}{d x_{i}}+\sum_{\alpha=1}^{m} \sum_{i_{1} \leqq \cdots \leqq i_{l}} \zeta_{\alpha}^{i_{1} \cdots i_{l}} \frac{\partial}{\partial p_{\alpha}{ }^{i_{1} \cdots i_{l}}}
$$

where

$$
\frac{d}{d x_{i}}=\frac{\partial}{\partial x_{i}}+\sum_{\alpha=1}^{m} p_{\alpha}{ }^{i} \frac{\partial}{\partial y_{\alpha}}+\sum_{q=1}^{l-1} \sum_{\alpha=1}^{m} \sum_{i_{1} \leq \cdots: i_{q}} p_{\alpha}^{i_{1} \cdots i_{q}{ }^{i}} \frac{\partial}{\partial p_{\alpha} i^{i_{1} \cdots i_{q}}}
$$

The element $E_{1}$ is an integral one of $\Sigma(\Phi)$ if and only if

$$
\sum_{i=1}^{n} \frac{d F}{d x_{i}} \lambda_{i}+\sum_{\alpha=1}^{m} \sum_{i_{1} \leqq \cdots \leqq i_{l}} \frac{\partial F}{\partial p_{\alpha}{ }^{i_{1} \cdots i_{l}}} \zeta_{\alpha}{ }^{i_{1} \cdots i_{l}}=0 \quad\left(F \in \Phi_{X}\right),
$$

where functions of $l$-jets are assumed to represent their values at $X$. The vector $\hat{\Gamma}$ of $T_{X}\left(J^{l}\right)$ possessing the form

$$
\widehat{\Gamma}=\sum_{i=1}^{n} \hat{\lambda}_{i} \frac{d}{d x_{i}}+\sum_{\alpha=1}^{m} \sum_{i_{1} \leqq \cdots \leqq i_{l}} \hat{\zeta}_{\alpha}^{i_{1} \cdots i_{l}} \frac{\partial}{\partial p_{\alpha}{ }^{i_{1} \cdots i_{l}}}
$$

belongs to $H\left(E_{1}\right)$ if and only if $\widehat{\Gamma}$ spanns a linear integral element and moreover

$$
\sum_{i=1}^{n}\left(-\zeta_{\alpha}^{i_{1} \cdots i_{l-1} i} \widehat{\lambda}_{i}+\widehat{\zeta}_{\alpha}^{i_{1} \cdots i_{l-1} i} \lambda_{i}\right)=0 \quad\left(1 \leqq \alpha \leqq m, 1 \leqq i_{1}, \cdots, i_{l-1} \leqq n\right)
$$

where the scalars $\zeta_{\alpha}^{i_{1} \cdots i_{l}}$ and the scalars $\widehat{\zeta}_{\alpha}{ }_{1_{1} \cdots i_{l}}$ are respectively supposed to be defined for all superscripts $\left\{i_{1}, \cdots, i_{l}\right\}$ in such a manner that they are respectively symmetric with respect to the superscripts $\left\{i_{1}, \cdots, i_{l}\right\}$. In fact, condition (14) means that the differential forms $\sum_{i=1}^{n} d p_{\alpha}{ }^{i_{1} \cdots i_{l-1} i} \wedge d x_{i}$ vanishes on the element spanned by $E_{1}$ and $\hat{\Gamma}$. 
Suppose that $\Phi$ is involutive at $X$. Then, according to Theorem B in $\S 4, \Sigma(\Phi)$ is involutive at $X$ with respect to $\Re$, and hence there exists at least one $n$-dimensional integral element $E_{n}$ of $\Sigma(\Phi)$ and of origin $X$ such that $\left(\rho_{-1}^{l}\right)_{*} E_{n}$ is of dimension $n$. Let $E_{n}$ be an $n$-dimensional subspace of $T_{X}\left(J^{l}\right)$ on which the contact forms of orders up to and including $l$ vanish. We know at once that the $E_{n}$ is spanned by $n$ vectors of the form

$$
\Gamma^{(i)}=\frac{d}{d x}+\sum_{\alpha=1}^{n} \sum_{i_{1} \sum_{i} \cdots i_{i}} \psi_{\alpha}^{i_{1} \cdots i_{l} i} \frac{\partial}{\partial p_{\alpha}^{i_{1} \cdots i_{l}}} \quad(i=1,2, \cdots, n) .
$$

In order that $E_{n}$ is an integral element of $\Sigma(\Phi)$, it is necessary and sufficient that

$$
\left\{\begin{array}{l}
\frac{d F}{d x_{i}}+\sum_{a=1}^{m} \sum_{i_{1} \leqq \cdots \leqq i_{l}} \psi_{\alpha}^{i_{1} \cdots i_{l} i} \frac{\partial F}{\partial p_{\alpha}^{i_{1} \cdots i_{l}}}=0 \quad\left(i=1,2, \cdots, n ; F \in \bar{\Phi}_{X}\right), \\
{\psi_{\alpha}{ }^{i_{1} \cdots i_{l+1}}=\psi_{\alpha}{ }^{k_{1} \cdots k_{l+1}}}_{\text {if }} \quad\left\{i_{1}, \cdots, i_{l+1}\right\}=\left\{k_{1}, \cdots, k_{l+1}\right\} .
\end{array}\right.
$$

Let $\mathfrak{P}$ be one of the prime ideals in Ass $(L(X) / M(X)), M(X)$ being the characteristic module of $\Phi$ at $X$. Recall that $\operatorname{Ass}(L(X) / M(X))$ coincides with the set $\left\{\beta_{1}, \cdots, \mathfrak{P}_{\nu}\right\}$ under the circumstances of Theorem III in the preceding section. We shall denote by $c(\mathfrak{P})$ the vector space of elements of degree 1 in $\mathfrak{P} ; c(\mathfrak{P})=\mathfrak{P} \cap R_{1}(X)$. Of course $c(\mathfrak{P})$ may possibly vanish.

Definition. We define the subspace $B(\mathfrak{S})$ of $T_{X}\left(J^{l}\right)$ to be the space spanned by all those vectors each of which is contained in an n-dimensional integral element $E_{n}$ of $\Sigma(\Phi)$ of origin $X$ such that dim $\left(\rho_{-1}^{l}\right)_{*} E_{n}=n$ and is also contained in $\left(\rho_{-1}^{l}\right)_{*}{ }^{-1} c(\mathfrak{P})$. We denote by $D(\mathfrak{F})$ the annihilator of $B(\mathfrak{F})$ in the dual space $T_{X}{ }^{*}\left(J^{l}\right)$ to $T_{X}\left(J^{l}\right)$.

The space $B(P)$ is expressed as follows:

$$
\begin{array}{r}
B(\mathfrak{F})=\left\langle\sum_{i=1}^{n} \lambda_{i}\left(\frac{d}{d x_{i}}+\sum_{\alpha=1}^{n} \sum_{i_{1} \leqq \cdots \leqq i_{l}} \psi_{\alpha}^{i_{1} \cdots i_{l} i} \frac{\partial}{\partial p_{\alpha}{ }^{i_{1} \cdots i_{l}}}\right) ;\right. \\
\left.\sum_{i=1}^{n} \lambda_{i} \frac{\partial}{\partial x_{i}} \in c(\mathfrak{P}),(16)\right\rangle,
\end{array}
$$

where by $\langle\cdots\rangle$ we mean the vector space over $K$ spanned by the elements in it. 
Let us investigate the structure of $B(\mathfrak{H})$ in detail. Let $\xi$ be an element of $T_{a}(\Re)$ (cf. $\S 4$ ). The linear mapping $\sigma(\xi)$ from $Q_{X}\left(J^{l}\right)$ $\otimes T_{a} *(\Re)$ to $Q_{X}\left(J^{l}\right)$ is defined to be the one obtained by extending linearly the mapping

$$
\sigma(\xi)\left(w \otimes v^{*}\right)=v^{*}(\xi) w, \text { where } w \in Q_{X}\left(J^{l}\right), \quad v^{*} \in T_{a}^{*}(\mathcal{\Omega}) .
$$

This mapping is expressed as follows: When $\xi=\sum_{i=1}^{n} \lambda_{i} \partial / \partial x_{i}$,

$$
\begin{aligned}
\sigma(\xi)\left(\sum_{i=1}^{n} \sum_{\alpha=1}^{m} \sum_{i_{1} \leqq \cdots \leq i_{l}} \varphi_{\alpha}^{i_{1} \cdots i_{l} i} \frac{\partial}{\partial p_{\alpha}^{i_{1} \cdots i_{l}}} \otimes d x_{i}\right) \\
=\sum_{\alpha=1}^{m} \sum_{i_{1} \leqq \cdots i_{l}}\left(\sum_{i=1}^{n} \lambda_{i} \varphi_{\alpha}^{i_{1} \cdots i_{l} i}\right) \frac{\partial}{\partial p_{\alpha}{ }^{i_{1} \cdots i_{l}}} .
\end{aligned}
$$

Since we have the expressions

$$
\begin{aligned}
& C_{X}(\Phi)=\left\{\sum_{\alpha=1}^{m} \sum_{i_{1} \sum_{\cdots} \cdots i_{l}} \varphi_{\alpha}^{i_{1} \cdots i_{l}} \frac{\partial}{\partial p_{\alpha}^{i_{1} \cdots i_{l}}} ;\right. \\
& \left.\sum_{\alpha=1}^{m} \sum_{i_{1} \leqq \cdots \leqq i_{l}} \varphi_{\alpha}^{i_{1} \cdots i_{l}} \frac{\partial F}{\partial p_{\alpha}^{i_{1} \cdots i_{l}}}=0 \quad\left(F \in \Phi_{X}\right)\right\}, \\
& p C_{X}(\Phi)=\left\{\sum_{\alpha=1}^{m} \sum_{i_{1} \leqq \cdots \leqq i_{l+1}} \varphi_{\alpha}^{i_{1} \cdots i_{l+1}} \frac{\partial}{\partial p_{\alpha}^{i_{1} \cdots i_{l+1}}} ;\right. \\
& \text { (17) } \left.\cdots \quad \sum_{\alpha=1}^{m} \sum_{i_{1} \leqq \cdots \leqq i_{l}} \varphi_{\alpha}^{i_{1} \cdots i_{l} i} \frac{\partial F}{\partial p_{\alpha} i_{1} \cdots i_{l}}=0 \quad\left(i=1,2, \cdots, n ; F \in \Phi_{X}\right)\right\} \text {, }
\end{aligned}
$$

where the same convention as in (14) is supposed to be applied, we know that $\sigma(\xi)$ induces the mapping from $p C_{X}(\Phi)$ to $C_{X}(\Phi)$.

Definition. Corresponding to each $\mathfrak{B} \in \operatorname{Ass}(L(X) / M(X))$, we introduce the following subspace of $C_{X}(\Phi)$ :

$$
C(\mathfrak{F})=\left\langle\sigma(\xi) p C_{X}(\Phi) ; \xi \in c(\mathfrak{P})\right\rangle .
$$

For simplicity, we write: $R=R(X), L=L(X), M=M(X)$. On account of Lemma 5 in $\S 3$, the annihilator of $C_{X}(\Phi)$ in $L_{l}$ is $M_{l}$ and that of $p C_{X}(\Phi)$ in $L_{l+1}$ coincides with $M_{l+1}$. Hence the dual spaces to $C_{X}(\Phi)$ and $p C_{X}(\Phi)$ are canonically identified with $L_{l} / M_{l}$ and $L_{l+1} / M_{l+1}$ respectively. Let $\sigma^{*}(\xi)$ be the adjoint mapping of $\sigma(\xi) ; \sigma^{*}(\xi): L_{l} / M_{l}$ $\rightarrow L_{l+1} / M_{l+1}$. It is easy to show that $\sigma^{*}(\xi)$ is given by $\sigma^{*}(\xi) \widetilde{z}=\xi \widetilde{z}$ 
$\left(\widetilde{z} \in L_{l} / M_{l}\right.$ ), the multiplication being the one when $L / M$ is regarded as a graded $R$-module. Moreover the kernel of $\sigma^{*}(\xi)$ coincides with the annihilator of the image of $\sigma(\xi)$. Therefore we know that the annihilator Ann $C(\mathfrak{P})$ of $C(\mathfrak{P})$ in $L_{l}$ is the intersection of the spaces $\left\{z \in L_{l}\right.$; $\left.\xi z \in M_{l+1}\right\}$, where $\xi$ ranges over all vectors in $c(\mathfrak{B})$. Consequently we have

$$
\text { Ann } C(\mathfrak{P})=\left\{z \in L_{l} ; c(\mathfrak{P}) z \subset M_{l+1}\right\}
$$

In case $\mathfrak{H}$ is generated by linear forms, that is, by $c(\mathfrak{P})$, the conditions $c(\mathfrak{P}) \quad z \subset M_{l+1}$ and $\mathfrak{P} z \subset M$ are equivalent. Hence we have the following

Lemma 7. The annihilator Ann $C(\mathfrak{B})$ of $C(\mathfrak{P})$ in $L_{l}$ is given by (18). If the ideal $\mathfrak{B}$ is generated by $c(\mathfrak{F})$, then

$$
\text { Ann } C(\mathfrak{P})=\left\{z \in L_{l} ; \mathfrak{\Re} z \subset M\right\} \text {. }
$$

Using the space $C(\mathfrak{P})$, we can decompose $B(\mathfrak{P})$ as follows.

Proposition 3. The space $B(\mathfrak{P})$ admits the following direct sum decomposition:

$$
B(\mathfrak{P})=\left\{E_{n} \cap\left(\rho_{-1}^{l}\right)_{*}^{-1} c(\mathfrak{P})\right\} \oplus C(\mathfrak{P}),
$$

where $E_{n}$ is an n-dimensional integral element of $\Sigma(\Phi)$ of origin $X$ satisfying $\operatorname{dim}\left(\rho_{-1}^{l}\right)_{*} E_{n}=n$.

Proof. Suppose that $E_{n}$ is spanned by (15). Let $\Gamma$ be any vector of $B(\mathfrak{P})$. It is expressed as follows:

$$
\Gamma=\sum_{i=1}^{n} \lambda_{i}\left(\frac{d}{d x_{i}}+\sum_{\alpha=1}^{m} \sum_{i_{1} \leqq \cdots i_{l}} \chi_{\alpha}^{i_{1} \cdots i_{l} i} \frac{\partial}{\partial p_{\alpha} i_{1} \cdots i_{l}}\right),
$$

where $\sum_{i=1}^{n} \lambda_{i} \partial / \partial x_{i} \in c(\mathfrak{B})$ and the scalars $\chi_{\alpha}{ }^{i_{1} \cdots i_{2} i}$ satisfy the same condition as the condition (16) satisfied by the $\psi_{\alpha}^{i_{1} \cdots i_{l} i}$. This vector possesses the expression $\Gamma=\Gamma_{1}+\Gamma_{2}$ where $\Gamma_{1}=\sum_{i=1}^{n} \lambda_{i} \Gamma^{(i)}$ and

$$
\Gamma_{2}=\sum_{a=1}^{m} \sum_{i_{1} \leqq \cdots \leqq i_{l}}\left\{\sum_{i=1}^{n} \lambda_{i}\left(\chi_{\alpha}^{i_{1} \cdots i_{l} i}-\psi_{\alpha}^{i_{1} \cdots i_{l} i}\right)\right\} \frac{\partial}{\partial p_{\alpha}^{i_{1} \cdots i_{l}}} .
$$


Obviously $\Gamma_{1}$ belongs to $E_{n}$. We assert that $\Gamma_{2}$ is actually a vector of $C(\mathfrak{P})$. In fact. the scalars $\varphi_{\alpha}^{i_{1} \cdots i_{l} i}=\chi_{\alpha}{ }^{i_{1} \cdots i_{l} i}-\psi_{\alpha}^{i_{1} \cdots i_{l} i}$ satisfy condition (17), and hence the vector

$$
\widetilde{\mathscr{X}}=\sum_{a=1}^{m} \sum_{i_{1} \leqq \cdots \leqq i_{l+1}} \varphi_{\alpha}^{i_{1} \cdots i_{l+1}} \frac{\partial}{\partial p_{\alpha}^{i_{1} \cdots i_{l+1}}}
$$

belongs to $p C_{X}(\Phi)$. Putting $\xi=\sum_{i=1}^{n} \lambda_{i} \partial / \partial x_{i} \in c(\mathfrak{F})$, we have $\Gamma_{2}=\sigma(\xi) \widetilde{\mathscr{X}}$. It follows from the very definition that $\Gamma_{2} \in C(\mathfrak{P})$. Thus the proof will be complete if we show that $C(\mathfrak{P})$ is a subspace of $B(\mathfrak{P})$. This fact is, however, proved without any difficulty.

Q.E.D.

The remaining part of this section is devoted to the investigation on characteristics in the sense of Cauchy and Cartan. Let $E_{p}$ be an integral element of origin $E_{0}$. The space $H\left(E_{p}\right)$ is obviously a subspace of $H\left(E_{0}\right)$. The element $E_{p}$ is called a Cauchy-Cartan characteristic element if and only if $H\left(E_{p}\right)$ coincides with $H\left(E_{0}\right)$ (cf. Cartan [2], Part VIII; Cartan [4], Chap. III; Goursat [7], §66). As is well known, Cauchy-Cartan characteristic elements can be characterized as an integral element of the characteristic system in the sense of Cartan. The Cartan characteristic system can be always constructed in the well-known manner, and hence one can know, at least in theory, whether or not a given differential system admits Cauchy-Cartan characteristics. There exists, however, the more explicit condition in order that a differential system admits Cauchy-Cartan characteristics. In fact, in the case of Pfaffian systems, Cartan [2] himself established such a condition stated by means of Cartan's characters. Owing to our theory developed above, we can establish such a new condition for the differential system $\Sigma(\bar{\Phi})$. We have actually the following

Theorem IV. Assume that the following two conditions are satisfied:

(i) $\Phi$ is involutive at $X$.

(ii) The characteristic module of $\Phi$ at $X$ itself is a $\mathfrak{P}$-primary submodule of $L=L(X)$ and its exponent is equal to 1.

Then the subspace $B(\mathfrak{P})$ of $T_{X}\left(J^{l}\right)$ is a $\gamma$-dimensional integral 
element of $\Sigma(\bar{D})$ such that $\operatorname{dim}\left(\rho_{-1}^{l}\right)_{*} B(\mathfrak{F})=\gamma$, where $\gamma=\operatorname{dim} c(\mathfrak{F})$. Moreover $B(\Im)$ is a Cauchy-Cartan characteristic element of $\Sigma(\Phi)$ posscssing the maximum dimension.

Proof. By virtue of Theorem B in $\S 4$, condition (i) implies that there exists an $n$-dimensional integral element $E_{n}$ of $\Sigma(\Phi)$ and of origin $X$ such that $\left(\rho_{-1}^{l}\right)_{*} E_{n}$ is of dimension $n$. In this proof, we denote by $M$ the characteristic module of $\Phi$ at $X$. We prove this theorem by dividing into four steps.

(a) $B(\mathfrak{S})=E_{n} \cap\left(\rho_{-1}^{l}\right)_{*}{ }^{-1} c(\mathfrak{F})$. In particular, $B(\mathfrak{K})$ is a $\gamma$-dimensional integral element.

In fact, on account of Proposition 3, it suffices to show that the space $C(\mathfrak{P})$ vanishes. Since the exponent of $M$ is equal to 1 by assumption, Lemma 7 indicates that the annihilator of $C(\mathfrak{Y})$ in $L_{l}$ coincides with the total space $L_{l}$. This means that $C(\mathfrak{P})$ vanishes.

(b) $H(X)=E_{n} \oplus C_{X}(\Phi)$ (direct sum decomposition).

Suppose $E_{n}$ is spanned by the $n$ vectors (15). Let $\Gamma$ be any vector of $H(X)$ expressed by (12). It is obvious that $\Gamma$ can be written in the following form:

$$
\Gamma=\sum_{i=1}^{n} \lambda_{i} \Gamma^{(i)}+\sum_{\alpha=1}^{m} \sum_{i_{1} \leqq \cdots \leqq i_{l}}\left(\zeta_{\alpha}^{i_{1} \cdots i_{l}}-\sum_{i=1}^{n} \lambda_{i} \psi_{\alpha}^{i_{1} \cdots i_{l} i}\right) \frac{\partial}{\partial p_{\alpha}{ }^{i_{1} \cdots i_{l}}} .
$$

The first term on the right hand side is obviously a rector of $E_{n}$. Since the $\zeta$ 's and the $\psi$ 's satisfy (13) and (16) respectively, we conclude at once that the second term is a vector in $C_{X}(\Phi)$. This completes the proof of (b).

(c) Let $E_{1}$ be a linear integral element of $\Sigma(\Phi)$ of orjgin $X$. In order that $E_{1}$ is contained in $H\left(E_{1}{ }^{\prime}\right)$ for any linear element $E_{1}{ }^{\prime}$ contained in $C_{Y}(\Phi)$, in other words, $H\left(E_{1}\right)$ contains $C_{Y}(\Phi)$, it is necessary and sufficient that $\left(\rho_{-1}^{l}\right)_{*} E_{1}$ is contained in $c(\mathfrak{P})$.

In fact, suppose that $E_{1}$ be spanned by $(12)$ and $E_{1}{ }^{\prime}$ be spanned by

$$
\mathcal{X}=\sum_{a=1}^{m} \sum_{i_{1} \leqq \cdots \leqq i_{l}} \varphi_{\alpha}^{i_{1} \cdots i_{l}} \frac{\partial}{\partial p_{\alpha}^{i_{1} \cdots i_{l}}}
$$

Then, $E_{1}$ is contained in $H\left(E_{1}^{\prime}\right)$ if and only if the following are satisfied (cf. (14)): 


$$
\sum_{i=1}^{n} \lambda_{i} \varphi_{\alpha}^{i_{1} \cdots i_{l-1} i}=0 \quad\left(1 \leqq \alpha \leqq m, 1 \leqq i_{1}, \cdots, i_{l-1} \leqq n\right),
$$

where the same convention as in (14) is assumed to be applied. Hence $E_{1}$ is contained in $H\left(E_{1}{ }^{\prime}\right)$ for all $E_{1}{ }^{\prime}$ contained in $C_{X}(\Phi)$ if and only if

$$
\sum_{i=1}^{n} \lambda_{i} \varphi_{\alpha}^{i_{1} \cdots i_{l-1} i} \equiv 0 \quad\left(\bmod \sum_{\alpha=1}^{m} \sum_{i_{1} \leqq \cdots \leq i_{l}} \frac{\partial F}{\partial p_{\alpha}^{i_{1} \cdots i_{l}}} \varphi_{\alpha}^{i_{1} \cdots i_{l}} ; F \in \Phi_{X}\right),
$$

where the $\varphi$ 's are regarded as indeterminates. We write $\xi_{i}=\partial / \partial x_{i}$ for brevity. Recalling Lemma 5 in $\S 3$, we find no difficulty in showing that the latter condition is equivalent to the condition

$$
\sum_{i=1}^{n} \lambda_{i} d y_{\alpha} \otimes \xi_{i_{1}} \bigotimes_{S} \cdots \bigotimes_{S} \xi_{i_{l-1}} \bigotimes_{S} \xi_{i_{l-1}} \in M_{l} \quad\left(\begin{array}{c}
\alpha=1,2, \cdots, m, \\
1 \leqq i_{1}, \cdots, i_{l-1} \leqq n
\end{array}\right) .
$$

Bearing in mind that the elements $d y_{\alpha} \otimes \xi_{i_{1}} \underset{S}{\bigotimes} \cdots \underset{S}{\bigotimes} \xi_{i_{l-1}}$ span $L_{l-1}$, we find immediately that this can be stated as follows:

$$
\left(\sum_{i=1}^{n} \lambda_{i} \xi_{i}\right) L_{l-1} \subset M_{l}
$$

When $M=L$, the theorem is obviously valid (In this case, $\Phi$ is actually completely integrable at $X$ ). In the contrary case, $M_{l-1}$ is a proper subspace of $L_{l-1}$ (cf. the very definition of the module associated with involutive subspaces in $\S 3$ ). In other words, there is an element of $L_{l-1}$ not belonging to $M$. Since $M$ is a $\mathfrak{P}$-primary submodule of $L$ with exponent 1, it follows that (20) holds if and only if $\sum_{i=1}^{n} \lambda_{i} \xi_{i}$ belongs to $\mathfrak{P}$, that is, to $c(\mathfrak{P})$. The latter condition means that $\left(\rho_{-1}^{l}\right)_{*} E_{1}$ is contained in $c(\mathfrak{P})$. The required result has been Therefore proved.

(d) We now complete the proof of Theorem IV. It suffices to prove the following:

"A linear element of origin $X$ is a Cauchy-Cartan characteristic element of $\Sigma(\Phi)$ if and only if it is contained in $B(\mathfrak{P})$."

Proof. Sufficiency: Suppose that $E_{1}$ is a linear element contained in $B(\mathfrak{P})$. By virtue of (a), $E_{1}$ is contained in the element $E_{n}$. Let $\Gamma^{\prime}$ be any vector of $H(X)$. By (b), $\Gamma^{\prime}$ admits the decomposition $\Gamma^{\prime}$ $=\Gamma_{1}{ }^{\prime}+\Gamma_{2}{ }^{\prime}$ where $\Gamma_{1}{ }^{\prime} \in E_{n}$ and $\Gamma_{2}{ }^{\prime} \in C_{X}(\Phi)$. Since $E_{1}$ and $\Gamma_{1}{ }^{\prime}$ is contained in the same integral element $E_{n}, \Gamma_{1}{ }^{\prime} \in H\left(E_{1}\right)$. Let $E_{1}{ }^{\prime}$ denote the integral element spanned by $\Gamma_{2}^{\prime}$. By virtue of $(\mathrm{a}),\left(\rho_{-1}^{l}\right)_{*} E_{1} \subset c(\mathfrak{P})$. 
Hence (c) implies that $H\left(E_{1}{ }^{\prime}\right) \supset E_{1}$, that is, $H\left(E_{1}\right) \supset E_{1}{ }^{\prime} \ni \Gamma_{2}{ }^{\prime}$. Combining what we have proved, we obtain $\Gamma^{\prime} \in H\left(E_{1}\right)$. This implies that $H\left(E_{1}\right)$ coincides with $H(X)$, and hence $E_{1}$ is actually a Cauchy-Cartan characteristic element.

Necessity: Suppose that $E_{1}$ is a Cauchy-Cartan characteristic element, that is, $H\left(E_{1}\right)=H(X)$. Then, it is necessary that $\left(\rho_{-1}^{l}\right)_{*} E_{1}$ is of dimension 1. In fact, if the contrary is true, that is, if $\left(\rho_{-1}^{l}\right)_{*} E_{1}=0$, then $E_{1}$ is contained in $C_{X}(\Phi)$ (cf. (b)). Assume $E_{1}$ is spanned by $\mathscr{X}$ given by (19). The integral element $E_{n}$ is obviously a subspace of $H\left(E_{1}\right)$, and hence any vector $\Gamma \in E_{n}$ belongs to $H\left(E_{1}\right)$. Therefore we have

$$
\sum_{i=1}^{n} \lambda_{i} \varphi_{a}^{i_{1} \cdots i_{l-1} i}=0 \quad\left(1 \leqq \alpha \leqq m, 1 \leqq i_{1}, \cdots, i_{l-1} \leqq n\right)
$$

for any system $\left(\lambda_{1}, \cdots, \lambda_{n}\right)$ of scalars (cf. (14)). This means that all the elements

$$
\sum_{i=1}^{n} \lambda_{i} d y_{\alpha} \otimes \xi_{i_{1}} \underset{S}{\otimes} \cdots \underset{S}{\otimes} \xi_{i_{l-1}} \bigotimes_{S} \dot{\xi}_{i} \quad\left(1 \leqq \alpha \leqq m, 1 \leqq i_{1}, \cdots, i_{l-1} \leqq n\right)
$$

annihilate $\mathscr{X}$. Since $\left(\lambda_{1}, \cdots, \lambda_{n}\right)$ is arbitrary, the elements (21) spanns $L_{l}$. Therefore $\mathfrak{X}$ must vanish. This is a contradiction, and hence $\left(\rho_{-1}^{l}\right)_{*} E_{1} \neq 0$.

By what we have just proved, we can choose an $(n-1)$-dimensional subspace $E_{n-1}$ of $E_{n}$ such that $\left(\rho_{-1}^{l}\right)_{*} E_{n-1}$ and $\left(\rho_{-1}^{l}\right)_{*} E_{1}$ spann the total space $T_{a}(\Re)$. The assumption $H\left(E_{1}\right)=H(X)$ implies that $E_{1}$ and $E_{n-1}$ spann an $n$-dimensional integral element $E_{n}{ }^{\prime}$ such that $\operatorname{dim}\left(\rho_{-1}^{l}\right)_{*} E_{n}{ }^{\prime}=n$. On the other hand, since $C_{X}(\Phi) \subset H(X)=H\left(E_{1}\right)$, (c) indicates that $\left(\rho_{-1}^{l}\right)_{*} E_{1}$ is contained in $c\left(\mathfrak{S}_{S}\right)$. Consequently $E_{1}$ in contained in $E_{n}{ }^{\prime}$ $\cap\left(\rho_{-1}^{l}\right) *^{-1} c(\mathfrak{B})$. By the very definition of $B(\mathfrak{P})$, it follows that $E_{1}$ is contained in $B($ S $)$. This completes the proof of necessity. Q.E.D.

\section{$\S 7$. Monge Characteristic Systems}

Let $X_{0}$ be an integral point of $\Phi$. Suppose that $\Phi$ is involutive at $X_{0}$. There exists a neighbourhood $C$ of $X_{0}$ in $I \Phi$ such that $\Phi$ is involutive at each point on $\varnothing$ and that the characters $s_{1}(X), \cdots, s_{n}(X)$ remains constant on $Q$ (cf. $\S \S 4-5)$. Let $M(X)$ be the characteristic module of $\Phi$ at $X \in C)$ and let it admit the reduced primary decomposition in $L(X)$ 


$$
M(X)=\bigcap_{j=1}^{\nu} Q_{j}(X) \quad(\nu=\nu(X)),
$$

where each $Q_{j}(X)$ is a $\mathfrak{F}_{j}(X)$-primary homogeneous submodule of $L(X)$, $\mathfrak{P}_{j}(X)$ being a homogeneous prime ideal in $R(X)$. We shall assume that the following regularity conditions (a), (b), and (c) are satisfied: (a) $\nu(X)$ remains constant on $C$. (b) Each subspace $c\left(\mathfrak{P}_{j}(X)\right)=\mathfrak{F}_{j}$. $(X) \cap R_{1}(X)$ of $T_{a}(\Re)\left(a=\rho_{-1}^{l} X\right)$ has the constant dimension $\gamma_{j}$ on $C$, and it depends analytically on $X \in C V$ in the sense that there exist $\gamma_{i}$ vector fields $\xi_{k}(X)=\sum_{i=1}^{n} a_{k i}(X) \partial / \partial x_{i}\left(1 \leqq k \leqq \gamma_{j}\right)$ where the functions $a_{k i}$ are analytic on $C$ such that their germs at $X$ spann the space $c\left(\mathfrak{P}_{j}(X)\right)$ for each $X$ on $C$. (c) The dimension of $C\left(\mathfrak{P}_{j}(X)\right)$ remains constant on $C$.

We shall denote by $\mathfrak{P}_{j}$ the ideal ${ }_{j}(X)$ considered as a field which assigns to each $X \in C$ the ideal $\mathfrak{P}_{j}(X)$. Let $\mathfrak{P}$ be one of $\mathfrak{F}_{1}, \cdots, \mathfrak{B}_{\text {y }}$ such that $c(\mathfrak{P}(X))$ is of positive dimension. For each $X \in \mathcal{V}, \mathfrak{P}$ defines a subspace $B\left(\mathscr{P}^{3}(X)\right)$ of $T_{X}\left(J^{l}\right)$ and its annihilator $D(\mathfrak{P}(X))$ in $L_{l}(X)$ (see $\S 6$ ). By Proposition 3 in $\S 6, B(\Re(X)$ ) is the direct sum of two subspaces $E_{n} \cap\left(\rho_{-1}^{l}\right)_{*}{ }^{-1} c(\mathfrak{P}(X))$ and $C(\mathfrak{P}(X))$. Therefore the space $B(\mathfrak{P}(X))$ has the constant dimension on $C V$. This fact enables us without any difficulty to prove that there exists an analytic Pfaffian system on CV such that the germs of Pfaffian forms belonging to it at $X$ spann the space $D(\mathfrak{P}(X))$ for each $X \in \mathcal{V}$. We shall denote the Pfaffian system by $D(\mathfrak{P})$.

Definition. The Monge characteristic system (of order l) of $\Phi$ defined on $C$ corresponding to $\supsetneqq$ is defined to be the Pfaffian system $D(\mathfrak{F})$. We shall denote it by $\Delta^{l}(\mathfrak{P})$.

Let us next define Monge characteristic systems of higher order. By virtue of Theorem $\mathrm{C}$ due to Cartan and Matsushima, the prolonged system $p^{q-l} \Phi$ is involutive at each point on $\left.C V^{q}=I\left(p^{q-l} \Phi\right) \cap\left(\rho_{l}{ }^{q}\right)^{-1} C\right)$ $(q \geqq l)$. Let $X^{q}$ be a point on $C V^{q}$ such that $\rho_{l}^{q} X^{q}=X$. It follows immediately from the definition that the characteristic module of $p^{q-l} \Phi$ at $X^{q}$ coincides with that of $\Phi$ at $X$. Hence we obtain the Monge characteristic system (of order $q$ ) of $p^{q-l} \Phi$ defined on $C V^{q}$ corresponding to $\mathfrak{B}$. 
Definition. The Monge characteristic system of order $q(\geqq l)$ of $\Phi$ over $C$ corresponding to $\Re$ is defined to be the Monge characteristic system of $p^{q-l} \Phi$ defined on $\mathcal{V}^{q}$ corresponding to $\mathfrak{F}$. We shall denote it by $\Delta^{q}(\mathfrak{P})$.

Let $u$ be a function of $q$-jets defined on a neighbourhood of $X^{q} \in C V^{q}$.

Definition. $u$ is called an integral of $\Delta^{q}(\mathfrak{B})$ at $X^{q}$ if and only if $d u \equiv 0\left(\bmod \Delta^{q}(\mathfrak{S} B)\right)$ on a neighbourhood of $X^{q}$ in $C^{q}$.

A function $u$ such that $d u \neq 0$ at $X^{q}$ is called a relative integral of $\Delta^{q}(\mathfrak{B})$ at $X^{q}$ if and only if $u$ vanishes at $X^{q}$ and

$$
d u \equiv 0\left(\bmod d^{q}(\mathfrak{P})\right) \text { on a neighbourhood of } X^{q} \text { in } C^{q}(u),
$$

where $C V^{q}(u)$ denotes the set of the points of $C V^{q}$ at which $u$ vanishes.

We find no difficulty in proving that a function of 4 -jets is an integral of $\Delta^{q}(\mathfrak{B})$ at $X^{q}$ if and only if it is an integral of $\Delta^{q+1}(\mathfrak{P})$ at each point on $C V^{q+1} \cap\left(\rho_{q}^{q+1}\right)^{-1} X^{q}$ (cf. Kakié [9], §5). Therefore we may say that a function is, or is not, an integral of a characteristic system without specifying the order. We shall denote by $\Delta(\mathfrak{P})$ the Monge characteristic system corresponding to $\mathfrak{P}$ without the specifying order. If a function of $q$-jets which cannot be regarded as a function of $(q-1)$-jets is a (relative) integral of $\mathfrak{J}^{q}(\mathfrak{P})$, we say that it is a (relative) integral of order $q$ of $1(\mathfrak{F})$.

Remark. E. Cartan carried out a very suggestive discussion concerning Monge characteristics ([4], Chap. IV, Part III). Our discussion clarifies, however, the structure of Monge characteristics of general nonlinear inrolutive systems much more completely than the classical theory.

\section{Part III. Involutive Systems Whose Characters of Order More Than One Vanish}

\section{§. Construction of New Involutive Subspaces}

The remaining sections are devoted to investigating involutive systems of which characters of order more than 1 vanish. Such systems are 
similar to systems with one unknown function of two independent variables. Suppose that $\Phi$ is involutive at $X$ and its characters $s_{2}(X), \cdots$, $s_{n}(X)$ vanish. By virtue of Theorem $\mathrm{A}$ in $\S 4$, the space $C_{X}(\Phi)$ is an involutive subspace of $Q_{\bar{X}}\left(J^{l-1}\right) \otimes T_{a} *(\Re)$. Moreover the latter assumption means that $g_{1}\left[C_{X}(\Phi)\right]=0$ (see $\S 5$ ). In this section, we treat the algebraic problem of constructing new involutive subspaces contained in such an involutive one. Instead of dealing with $C_{Y}(\Phi)$ itself, we find it appropriate to consider any subspace of $Q_{X}\left(J^{l}\right)$. Throughout this section, by involutive subspaces we mean involutive subspaces of $Q_{\bar{X}}\left(J^{l-1}\right)$ $\otimes T_{a}^{*}(\Re)$. For brevity we shall write: $R=R(X), L=L(X)$ (see $\S 5$ ).

The following lemma is an immediate consequence of the definition (see $\S 2)$.

Lemma 8. Let $A$ be a subspace of $Q_{X}\left(J^{l}\right)$ such that $g_{1}[A]=0$. Then $A$ is an involutive subspace if and only if $\operatorname{dim} p A=\operatorname{dim} A$.

Suppose that $A$ is an involutive subspace in $Q_{X}\left(J^{l}\right)$ satisfying $g_{1}[A]$ $=0$. Associated with $A$ we have the submodule $M(A)$ of $L$ (the module associated with $A$; see $\S 3$ ). Except when any confusion occurs, we shall write: $M=M(A)$. Let $M=\bigcap_{j=1}^{\nu} Q_{j}$ be a reduced primary decomposition of $M$ in $L$ where each $Q_{j}$ is a $\mathfrak{P}_{j}$-primary homogeneous submodule of $L$. No $\mathfrak{P}_{j}$ coincides with the maximal ideal $\mathfrak{X}$ in $R$ (see $\S 3$ ). By Lemma 6 in $\S 3$, the homogeneous prime ideals $\mathfrak{P}_{j}$ in $R$ are of projective dimension 0 . The fact that $\mathfrak{P}_{j}$ is generated by an $(n-1)$-dimensional subspace of $R_{1}$ means that the zero of $\mathfrak{P}_{j}$ is located in the $(n-1)$-dimensional projective space $P^{n-1}(K)$ over $K$.

Let $Z$ be a subset of $L_{l}$. We shall denote by $A[Z]$ the subspace of $A$ whose annihilator in $L_{l}$ is the space spanned by $M_{l}$ and $Z$. In particular, $A[0]=M_{l}$ (Lemma 5 in $\S 3$ ).

Lemma 9. Assume that $A$ is an involutive subspace satisfying $g_{1}[A]=0$. Let $z$ be an element of $L_{l}$ not belonging to $M_{l}$. Then $A[z]$ is an involutive subspace if and only if the following two equivalent conditions are satisfied:

(a) There exist $n-1$ independent linear forms $\xi_{1}, \cdots, \xi_{n-1}$ in $R$ such that $\xi_{1} z, \cdots, \xi_{n-1} z \in M_{l+1}$. 
(b) There exists a prime ideal $\mathfrak{P}$ belonging to $\operatorname{Ass}(L / M)$ such that it is generated by $n-1$ independent linear forms and that $\mathfrak{P z} \subset M$.

Proof. We write: $A^{\prime}=A[z]$. Obviously $g_{1}\left[A^{\prime}\right]=0$. By Lemma $8, A^{\prime}$ is an involutive subspace if and only if $\operatorname{dim} p A^{\prime}=\operatorname{dim} A^{\prime}$. Since $\operatorname{dim} p A=\operatorname{dim} A$, we readily find that $A^{\prime}$ is an involutive subspace if and only if

$$
\operatorname{dim}\left\langle R_{1} z, R_{1} M_{l}\right\rangle / R_{1} M_{l}=1 .
$$

We remark that $R_{1} z$ is not contained in $R_{1} M_{l}=M_{l+1}$. In fact, if the contrary is valid, we have $R_{1} z \subset M=\bigcap_{j=1}^{\nu} Q_{j}$. Clearly there is $\xi \in R_{1}$ not belonging to $\bigcup_{j=1}^{\nu} \mathfrak{P}_{j}$. Therefore, using the property of primary submodules, we can readily show that $z \in M$. This contradicts the assumption. Thus we have proved the above remark. Bearing in mind this fact, we find no difficulty in proving that condition (22) holds if and only if condition (a) holds, and hence $A^{\prime}$ is an involutive subspace if and only if (a) holds.

Let us prove that the conditions (a) and (b) are equivalent. Suppose that (a) valid. Let $\mathfrak{P}$ be the prime ideal generated by $\xi_{1}, \cdots, \xi_{n-1}$. Then $\mathfrak{P} z \subset M$. Since $z \notin M$, this implies that $\mathfrak{B} \subset \mathfrak{x}_{L}(M)=\bigcap_{j=1}^{\nu} \mathfrak{P}_{j}$ (cf. $\S 1$ ). Hence there is a prime ideal $\mathfrak{P}_{k}$ such that $\mathfrak{B} \subset \mathfrak{B}_{k}$. Remarking that both ideals have the same projective dimension 0 , we have $\mathfrak{P}=\mathfrak{P}_{k}$. Thus (a) implies (b). Suppose conversely that (b) is valid. Let $\mathfrak{P}$ be generated by $\xi_{1}, \cdots, \xi_{n-1}$. Then it is obvious that for the $\xi_{i}$, the condition in (a) is satisfied. Thus (b) implies (a). Q.E.D.

Corresponding to each $\mathfrak{P}_{\beta} \in \operatorname{Ass}(L / M)$, we have a subspace $C\left(\mathfrak{P}_{\beta}\right)$ of $A$ (see $\S 6)$ :

$$
C\left(\mathfrak{P}_{\beta}\right)=\left\langle\sigma(\xi) p A ; \xi \in c\left(\mathfrak{P}_{\beta}\right)\right\rangle .
$$

(Except in this section, we denote by the same symbol $C\left(\mathfrak{P}_{\beta}\right)$ the space constructed from the space $A=C_{X}(\Phi)$.) By Lemma 7 in $\S 6$, the annihilator of $C\left(\mathfrak{P}_{\beta}\right)$ in $L_{l}$ is $\left\{z \in L_{l} ; c\left(\mathfrak{P}_{\beta}\right) z \subset M\right\}$. Suppose that $\mathfrak{P}_{\beta}$ is generated by $n-1$ independent linear forms, that is, it is generated by 
$c\left(\mathfrak{F}_{\beta}\right)$. Then

$$
\text { Ann } C\left(\mathfrak{P}_{\beta}\right)=\left\{z \in L_{l} ; \mathfrak{P}_{\beta} z \subset M\right\} \text {. }
$$

We now modify Lemma 9 as follows.

Proposition 4. Suppose that $A$ is an involutive subspace satisfying $g_{1}[A]=0$. Let $A^{\prime}$ be a subspace of $A$ and of codimension 1 in $A$. Then in order that $A^{\prime}$ is an involutive subspace, it is necessary and sufficient that there exists a prime ideal $\mathfrak{P}_{\beta}$ in $\operatorname{Ass}(L / M)$ of which zero is located in $P^{n-1}(K)$ such that $A^{\prime}$ contains $C\left(\mathfrak{P}_{\beta}\right)$.

Proof. Assume $A^{\prime}$ is given by $A[z]\left(z \in L_{l}\right)$. By Lemma 9, $A^{\prime}$ is an involutive subspace if and only if there exists an ideal $\mathfrak{P}_{\beta}$ in Ass $(L / M)$ of which zero is located in $P^{n-1}(K)$ such that $\mathfrak{P}_{\beta} z \subset M$. The remark made above indicates that $\mathfrak{H}_{\beta} z \subset M$ if and only if $z \in$ Ann $C\left(\mathfrak{S}_{\beta}\right)$. The latter means that $A[z] \supset C\left(\mathfrak{P}_{\beta}\right)$. Consequently we have the desired result.

Q.E.D.

Let us generalize Proposition 4.

Proposition 5. Assume that $A$ is an involutive subspace satisfying $g_{1}[A]=0$. Let $\mathfrak{P}_{\beta}$ be an ideal in $\operatorname{Ass}(L / M)$ whose zero is located in $P^{n-1}(K)$. Then any subspace $A^{\prime}$ of $A$ containing $C\left(\mathfrak{P}_{\beta}\right)$ is an involutive one. Moreover the module $M\left(A^{\prime}\right)$ associated with $A^{\prime}$ admits the primary decomposition in L of the following form: $M\left(A^{\prime}\right)$ $=N \cap\left\{\bigcap_{j \neq \beta} Q_{j}\right\}$ where $N$ is either the total module or a $\mathfrak{i}_{3}$-primary submodule containing $Q_{\beta}$ such that its multiplicity $\mu(N ; L)$ is less than $\|\left(Q_{\beta} ; L\right)$ by the codimension of $A^{\prime}$ in $A$.

Proof. Let $s$ denote the codimension of $A^{\prime}$ in $A$. Suppose that $A^{\prime}=A\left[z_{1}, \cdots, z_{s}\right]$ where $z_{1}, \cdots, z_{s} \in L_{l}$. On account of Lemma 7 in $\S 6$, the assumption $A^{\prime} \supset C\left(\mathfrak{F}_{\beta}\right)$ implies $\mathfrak{P}_{\beta} z_{i} \subset M(1 \leqq i \leqq s)$. Remarking that $\mathfrak{P}_{\beta}$ is generated by $n-1$ independent linear forms, we conclude from this that

$$
\operatorname{dim}^{\prime}, M_{l+1}, R_{1} z_{i} ; 1 \leqq i \leqq s>/ M_{l+1} \leqq s
$$


In this inequality, the equality is actually valid. In fact, suppose that the numbe on the left side is less than $s$. Then there is a linear combination $z=\sum_{j=1}^{s} c_{i} z_{i}$ with $c_{i} \in K$ such that $R_{1} z \subset M_{l+1}$. This implies that $z \in M_{l}$ (recall the property of the module associated with an involutive subspace; see $\S 3$ ). This is a contradiction to the choice of the elements $z_{i}$. Thus the equality holds. This equality implies that $\operatorname{dim} p A^{\prime}=\operatorname{dim}$ $A^{\prime}$. Hence, by Lemma $8, A^{\prime}$ is an involutive subspace.

We next prove the latter assertion. Let $M\left(A^{\prime}\right)=\bigcap_{k=1}^{r} N_{(k)}$ be a reduced primary decomposition where $N_{k^{*}}$ is an $\mathfrak{P}_{k}$-primary submodule of $L$. No $\mathfrak{U}_{k}$ is the maximal ideal $\mathfrak{X}$ in $R$. Since $g_{1}\left[A^{\prime}\right]=0$, on account of Lemma 6 in $\S 3$, the homogeneous prime ideals $\mathfrak{A}_{k}$ are of projective dimension 0. Obviously $M\left(A^{\prime}\right) \supset M$, and hence $N_{(k)} \supset \bigcap_{j=1}^{\nu} Q_{j}(1 \leqq k \leqq r)$. Taking the operation $\mathfrak{r}_{L}\left(\quad\right.$ ) (see $\S 1$ ), we get $\mathfrak{N}_{k} \supset \bigcap_{j=1}^{\nu} \mathfrak{P}_{j}$. From this it follows that each $\mathfrak{P L}_{k}$ contains one of the ideals $\mathfrak{Y}_{j}$. Since both ideals have the same dimension, they must coincide. We may assume without loss of generality that $\mathfrak{P}_{k}=\mathfrak{P}_{k}(1 \leqq k \leqq r)$. The prime ideal $\mathfrak{A}_{k}=\mathfrak{F}_{k}$ does not contain $\mathfrak{F}_{j}$ for $j \neq k$. We prove that the facts $N_{(k)} \supset \bigcap_{j=1}^{\nu} Q_{j}, \mathfrak{A}_{k} \not \mathfrak{F}_{j}$ for each $j \neq k$ implies that $N_{(k)} \supset Q_{k}$. In fact, let $q$ be an integer in $\{1, \cdots, \nu\}$ distinct from $k$. We first prove that $N_{(k)} \supset \underset{j \neq q}{\bigcap} Q_{j}$. To show this, it is sufficient to prove that any element $z$ of $\cap Q_{j \neq q}$ not belonging to $Q_{q}$ belongs to $N_{(k)}$. By assumption there is an element $\xi$ of $\mathfrak{P}_{q}$ not belonging to $\mathfrak{U}_{k}$. There is a positive integer $s$ such that $\xi^{s} z \in Q_{q}$. Hence $\xi^{s} z \in \bigcap_{j=1}^{\nu} Q_{j} \subset N_{(k)}$. Since the homomorphism from $L / N_{(k)}$ into itself defined by $\xi \notin \mathfrak{A}_{k}$ is an isomorphism (cf. The definition of primary submodules), we have $z \in N_{(k)}$. By the same reasoning, we have $N_{(k)} \supset \underset{j \neq q, t}{ } Q_{j}$ where $q \neq k, t \neq k$. Proceed step by step, we finally obtain $N_{(k)} \supset Q_{k}$.

Thus we have proved that $N_{(k)} \supset Q_{k}(1 \leqq k \leqq r)$.

To simplify the explanation, without loss of generality, we assume $\beta=\nu$. Then

$$
\text { Ann } A^{\prime} \subset \text { Ann } C\left(\mathfrak{F}_{\nu}\right)=\left\{z \in L_{l} ; \mathfrak{\beta}_{\nu} z \subset M\right\} \text {. }
$$

Since $\mathfrak{F}_{\nu}$ does not contain $\mathfrak{H}_{j}$ for $j \neq \beta, \mathfrak{S}_{\nu} z \subset M=\bigcap_{j=1}^{\nu} Q_{j}$ implies that $z \in \bigcap_{j \neq \nu} Q_{j}$ (cf. the above argument). Hence Ann $A^{\prime}$ is contained in 
$\left\{\bigcap_{j \neq \nu} Q_{j}\right\} \cap L_{l}$. It follows that $M\left(A^{\prime}\right)$ is contained in $H\left(\bigcap_{j \neq \nu} Q_{j}\right)=\bigcap_{j=1}^{\nu-1} Q_{j}$ (see $\S 3$ ). By the same reasoning as we have just done, we know that each $\mathfrak{P}_{j}(1 \leqq j \leqq \nu-1)$ coincides with one of the ideals $\mathfrak{U}_{k}$ and that if $\mathfrak{P}_{j}=\mathfrak{U}_{k}$, then $Q_{j} \supset N_{(k)}$. Combining this with the above result, we conclude that the number $r$ is equal to either $\nu-1$ or $\nu$, and that $N_{(k)}$ $=Q_{k}(1 \leqq k \leqq \nu-1)$. When $r=\nu-1$, the proof has been already completed. When $r=\nu$, we have already proved that $\mathfrak{A}_{\nu}=\mathfrak{P}_{\nu}$ and $N_{(\nu)} \supset Q_{\nu}$. Therefore, in any case, we have the primary decomposition of $M\left(A^{\prime}\right)$ stated in the proposition. Moreover, applying Theorem II in $\S 3$, we have

$$
\sum_{j=1}^{\nu} \mu\left(Q_{j} ; L\right)=\operatorname{dim} A, \mu(N ; L)+\sum_{j=1}^{\nu-1} \mu\left(Q_{j} ; L\right)=\operatorname{dim} A^{\prime} .
$$

Hence $\mu(N ; L)=\mu\left(Q_{\nu} ; L\right)-\left(\operatorname{dim} A-\operatorname{dim} A^{\prime}\right)$. Thus we have proved all the required results.

Q.E.D.

Proposition 6. Suppose that $A$ is an involutive subspace satisfying $g_{1}[A]=0$. Let $\mathfrak{P}_{1}, \cdots, \mathfrak{P}_{s}$ be $s$ prime ideals in $\mathrm{Ass}(L / M)$ such that their zeros are located in $P^{n-1}(K)$. Let $A_{k}$ be any subspace of $A$ containing $C\left(\mathfrak{P}_{k}\right)(1 \leqq k \leqq s)$. Then the space $\bigcap_{k=1}^{s} A_{k}$ is an involutive subspace whose codimension in $A$ is equal to the sum of the codimensions in $A$ of the s spaces $A_{k}$. Moreover the module associated with $\bigcap_{k=1}^{s} A_{k}$ admits the primary decomposition $\left\{\bigcap_{k=1}^{s} N_{(k)}\right\} \cap\left\{\bigcap_{j=s+1}^{\nu} Q_{j}\right\}$ where each $N_{(k)}$ is either the total module or a $\mathfrak{B}_{k}$-primary submodule of $L$.

Proof. We write: $B_{0}=A, B_{k}=\bigcap_{j=1}^{k} A_{j}(1 \leqq k \leqq s)$. By assumption, $B_{0}$ is an involutive subspace. By Proposition $5, B_{1}$ is also an involutive subspace and its associated module $M\left(B_{1}\right)$ admits the primary decomposition $N_{(1)} \cap\left\{\bigcap_{j=2}^{\nu} Q_{j}\right\}$ where $N_{(1)}$ is either the total module or a $\mathfrak{B}_{1}$-primary submodule. We note that $B_{2}$ is a subspace of the involutive subspace $B_{1}$ constructed from $B_{1}$ exactly in the same way as $B_{1}$ is constructed from $A$. In fact, the space $C\left(\mathfrak{P}_{j}\right)$ constructed from $A$ contains the corresponding space constructed from $B_{1}=A_{1}$ as is immediately seen. Therefore we can apply Proposition 5 again, and we conclude that $B_{2}$ is an involutive 
subspace and $M\left(B_{2}\right)$ admits the primary decomposition $N_{(1)} \cap N_{(2)} \cap\left\{\bigcap_{j=3}^{\nu} Q_{j}\right\}$ where $N_{(2)}$ is either the total module or a $\mathfrak{P}_{2}$-primary submodule. Proceeding step by step in the same way, we see that the subspaces $B_{k}$ are involutive ones. In particular, $\bigcap_{j=1}^{\nu} A_{j}$ is indeed an involutive subspace.

Let us prove the assertion concerning the codimension of that space. We prove that $\operatorname{dim} B_{k} / B_{k+1}$ is equal to the codimension $\gamma$ of $A_{k+1}$ in $A(1 \leqq k<s)$. Suppose $A_{k+1}$ is given by $A\left[z_{1}, \cdots, z_{r}\right]$ where $z_{i} \in L_{l}$. It follows from $C\left(\mathfrak{P}_{k+1}\right) \subset A_{k+1}$ that $\mathfrak{P}_{k+1} z_{i} \subset M(1 \leqq i \leqq \gamma)$. This implies $z_{i} \in \bigcap_{j \neq k+1} Q_{j}$ (cf. the argument in the proof of Proposition 5). Since $z_{i} \notin M$, $z_{i} \notin Q_{k-1}$. Hence $z_{i} \notin$ Ann $B_{k}=M\left(B_{k}\right) \cap L_{l}$ (cf. Lemma 5 in $\S 3$ ), for Ann $B_{k}$ is contained in $Q_{k+1} \cap L_{l}$. From this it follows that $B_{k+1}=B_{k}$ $\cap A_{k+1}$ is of codimension $\gamma$ in $B_{k}$.

Using what we have just deduced, we can readily calculate the codimension of $B_{s}$, and we know that the assertion is indeed true.

Q.E.D.

In the above proof, if the exponents of $Q_{k}(1 \leqq k \leqq s)$ in $L$ are equal to 1 , Ann $C\left(\mathfrak{P}_{k}\right)$ coincides with $\left\{\bigcap_{j \neq k} Q_{j}\right\} \cap L_{l}$. In fact, by the same argument as in the proof of Proposition 5, we can prove the following:

$$
\left\{z \in L ; \Re_{k} z \subset M\right\}=\bigcap_{j \neq k} Q_{j} .
$$

Hence we have the desired result. Accordingly when $A_{k}=C\left(\mathfrak{P}_{k}\right) \quad(1 \leqq k$ $\leqq s)$, we readily see that the submodule $N_{(k)}$ must coincide with the total module (note that the modules associated with involutive subspaces coincides if and only if their homogeneous part of degree $l$ coincides; see $\S 3$ ). Hence we have the following

Corollary. Under the circumstances of Proposition 6, suppose further that the exponents of $Q_{k}(1 \leqq k \leqq s)$ in $L$ is equal to 1 . Then the module associated reith the involutive subspace $\bigcap_{k=1}^{s} C\left(\mathfrak{F}_{k}\right)$ admits the reduced primary decomposition $\bigcap_{j=s+1}^{\nu} Q_{j}$.

In concluding this section, we calculate the codimension of $C\left(\mathfrak{P}_{\beta}\right)$ in $A$. 
Lemma 10. Suppose that $A$ is an involutive subspace such that $g_{1}[A]=0$. Let $\mathfrak{F}_{\beta}$ be an ideal in $\operatorname{Ass}(L / M)$ such that the zero of $\mathfrak{P}_{\beta}$ is located in $P^{n-1}(K)$. If the exponent of $Q_{\beta}$ in $L$ is equal to 1 , the codimension of $C\left(\mathfrak{P}_{\beta}\right)$ in $A$ is equal to $\mu\left(Q_{\beta} ; L\right)$.

Proof. By Proposition 6 and its corollary, $C\left(\mathfrak{F}_{\beta}\right)$ is an involutive subspace and the module associated with it admits the reduced primary decomposition $\bigcap_{j \neq \beta} Q_{j}$. Since the prolongation of involutive subspaces are also involutive ones (cf. Note at the end of $\S 2$ ), applying Lemma 8 successively, we have

$$
\operatorname{dim} A=\operatorname{dim} p^{i} A, \quad \operatorname{dim} C\left(\mathfrak{P}_{\beta}\right)=\operatorname{dim} p^{i} C\left(\mathfrak{P}_{\beta}\right) \text { for } i \geqq 0 .
$$

By virtue of Lemma 5 in $\S 3$ and Lemma 6.1 in Kuranishi's work [12], the annihilators of $p^{i} A$ and $p^{i} C\left(\mathfrak{S}_{\beta}\right)$ are $M_{l+i}$ and $\left\{\bigcap_{j \neq \beta} Q_{j}\right\} \cap L_{l+i}$ respectively. Accordingly the codimension of $C\left(\mathfrak{P}_{\beta}\right)$ in $A$ can be known by calculating the dimensions of the latter spaces.

Since the prime ideals $\mathfrak{P}_{j}$ are distinct and of projective dimension 0 , it is not difficult to show that the space $\left(\mathfrak{P}_{\beta}+\bigcap_{j \neq \beta} \mathfrak{P}_{j}{ }^{\sigma}\right) \cap R_{q}$ coincides with the total space $R_{q}$ for large $q$, where $\sigma$ is a positive integer. Since $\mathfrak{P}_{j}{ }^{\sigma} L \subset Q_{j}(1 \leqq j \leqq \nu)$ for large $\sigma$, we know at once that $\left(Q_{\beta}+\bigcap_{j \neq \beta} Q_{j}\right) \cap L_{q}$ $=L_{q}$ for large $q$. Let us prove the vector space isomorphism

$$
\left(\bigcap_{j \neq \beta} Q_{j}\right) \cap L_{q} / M_{q} \cong L_{q} / Q_{\beta} \cap L_{q} .
$$

Consider the natural mapping $\tau$ from $\left(\bigcap_{j \neq \beta} Q_{j}\right) \cap L_{q}$ to the space on the right side. By the above fact, $\tau$ is actually surjective. Since the kernel of $\tau$ is obviously $M_{q}$, we get the desired isomorphism.

Combining what we have shown, we find that the codimension of $C\left(\mathfrak{P}_{\beta}\right)$ in $A$ is equal to $\operatorname{dim} L_{q} / Q_{\beta} \cap L_{q}$ for large $q$. It follows from the definition of $\mu\left(Q_{\beta} ; L\right)$ that the latter is equal to $\mu\left(Q_{\beta} ; L\right)$ for large $q$, and hence we have the desired result.

Q.E.D.

\section{§9. Construction of New Involutive Systems}

The problem considered in this section is to construct new involutive systems of which solutions are also those of a given one. One of the 
essential problems to be solved in treating this problem is the algebraic one of constructing new involutive subspaces contained in a given one. In the preceding section, we have solved the latter problem only for involutive subspaces associated with involutive systems whose characters of order more than 1 vanish. In the general case, we find pretty different circumstances in solving this problem. It is on account of this situation that we shall deal with only such systems.

Suppose that $\Phi$ satisfies the following condition:

$(\mathrm{H}-1) \Phi$ is involutive at $X_{0}$ and $s_{1}\left(X_{0}\right)>0, s_{2}\left(X_{0}\right)=\cdots=s_{n}\left(X_{0}\right)=0$.

Then there is a neighbourhood $\varnothing$ of $X_{0}$ in $I \Phi$ such that the condition $(\mathrm{H}-1)$ is satisfied at each point on $C)$ and the character $s_{1}(X)$ remains constant on $C V$ (cf. $\S \S 4-5$ ). Let the characteristic module $M(X)$ of $\Phi$ at $X \in C$ admit the reduced primary decomposition in $L(X)$

$$
M(X)=Q_{1}(X) \cap Q_{2}(X) \cap \cdots \cap Q_{\nu}(X) \quad(\nu=\nu(X))
$$

where $Q_{j}(X)$ is a $\mathfrak{P}_{j}(X)$-primary homogeneous submodule of $L(X)$, $\mathfrak{P}_{j}(X)$ being a homogeneous prime ideal in $R(X)$. For simplicity we write: $\mu_{j}(X)=\mu\left(Q_{j}(X) ; L(X)\right)$. In virtue of Theorem III in $\S 5$, the ideals $\Re_{j}(X)$ are of projective dimension 0 and moreover $\sum_{j=1}^{\nu} \mu_{j}(X)=s_{1}$. $(X)$. We shall furthermore assume the following condition:

$(\mathrm{H}-2)$ There exists a neighbourhood $C$ of $X_{0}$ in $I \Phi$ such that the following are satisfied: (a) The number $\nu(X)$ remains constant on $C$ and the exponents of the submodules $Q_{j}(X)$ in $L(X)$ are equal to 1 . (b) Each ideal $\mathfrak{P}_{j}(X)$ is generated by an $(n-1)$-dimensional subspace of $R_{1}(X)$ and it is analytic in the sense that there exist $n-1$ vector fields $\xi_{k}(X)=\sum_{i=1}^{n} a_{k i}(X) \partial / \partial x_{i}(k=1,2, \cdots, n-1)$ where the functions $a_{k i}(X)$ are analytic on $C$ such that the germs of the vector fields $\xi_{k}(X)$ at $X$ generate the ideal $\mathfrak{P}_{j}(X)$ for each $X \in \mathcal{C}$.

Applying Lemma 10 at the end of $\S 8$, we know that $\operatorname{dim} C\left(9_{j}\right)$ is equal to $g_{0}(X)-\mu_{j}(X) \quad\left(g_{0}(X)=\operatorname{dim} C_{X}(\varpi)\right)$. Since the functions $\mu_{j}(X)$ are lower semi-continuous and $s_{1}(X)=g_{0}(X)$ is constant on $C$, the equality $\sum_{j=1}^{\nu} \mu_{j}(X)=s_{1}(X)$ implies that the numbers $\mu_{j}(X)$ remain constant on $C V$. Therefore condition (c) stated at the beginning of $\S 8$ is satisfied, and hence we can define Monge characteristic systems $\Delta^{n}\left(\mathfrak{P}_{j}\right)$ which are actually analytic Pfaffian systems on $W$ (see $\S 8$ ). Hereafter 
we shall denote briefly by $s_{1}$ and $\mu_{j}$ respectively the numbers $s_{1}(X)$ and $u_{j}(X)$ being constant on $C V$.

Let $u_{1}, \cdots, u_{r}$ be $r$ functions defined on an open set $U_{1}$ in $J^{h_{1}}, \cdots$, on an open set $U_{r}$ in $J^{h_{r}}$ respectively $\left(h_{1}, \cdots, h_{r} \geqq l\right)$. We shall write: $h$ $=\max \left\{h_{i} ; 1 \leqq i \leqq r\right\}, \quad \widetilde{U}=\bigcap_{i=1}^{r}\left(\rho_{h_{i}}^{h}\right)^{-1} U_{i}$. Let $\left(u_{i}\right)$ denote the subsheaf of ideals in the sheaf $\mathcal{O}\left(U_{i}\right)$ of rings of germs of analytic functions on $U_{i}$ associated with the presheaf $\left\{\mathscr{F}_{u^{\prime}} ; U^{\prime} \subset \mathcal{U}_{i}\right\}$ with natural restriction mappings, where $\mathscr{F} u^{\prime}$ denotes the ideal generated by $u_{i}$ in the ring of analytic functions on $\mathcal{U}^{\prime}$. Let $\mathcal{H}_{u^{*}}$ be that ideal in the ring of analytic functions on $U^{*}$ which is generated by all sections of the sheaves $p^{h-l} \Phi$, $p^{h-h_{1}}\left(u_{1}\right), \cdots, p^{h-h_{r}}\left(u_{r}\right)$. We denote by $\Phi^{h}\left(u_{1}, \cdots, u_{r}\right)$ the sheaf of ideals

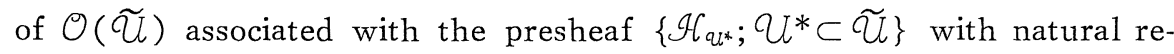
striction mappings. $\Phi^{h}\left(u_{1}, \cdots, u_{r}\right)$ is a system of partial differential equations of order $h$ with domain $\widetilde{U}$ such that its solutions are also those of $\Phi$.

Let $u$ be a function of $q$-jets defined on a neighbourhood of $X^{q}$ in $J^{q}(q \geqq l)$. We write:

$$
\pi_{q}^{*} u=\sum_{a=1}^{m} \sum_{i_{1} \leqq \cdots \leqq i_{q}} \frac{\partial u}{\partial p_{\alpha}^{i_{1} \cdots i_{q}}}\left(X^{q}\right) d y_{\alpha} \otimes \frac{\partial}{\partial x_{i_{1}}} \otimes \cdots \otimes \bigotimes_{S} \frac{\partial}{\partial x_{i_{q}}},
$$

which is an element of $L_{q}=Q_{b}(\mathscr{M}) \otimes S^{q}\left(T_{a} *(\Re)\right)$. We say that $u$ is independent of $\Phi$ at $X^{q}$ if and only if

$$
\pi_{q}^{*} u \neq 0\left(\bmod \pi_{q}^{*} F ; F \in\left(p^{q-l} \bar{\Phi}\right)_{X q}\right) .
$$

When $\Phi$ is involutive at $X=\rho_{l}{ }^{q} X^{q} \in C$, this condition is equivalent to the condition $\pi_{q}^{*} u \notin M_{q}(X)$ (cf. Lemma 5 in $\S 3$ ).

We are now in a position to discuss the problem proposed at the beginning of this section.

Proposition 7. Suppose that $\bar{D}$ satisfies the conditions $(\mathrm{H}-1)$ and $(\mathrm{H}-2)$. Let $u$ be a function of l-jets defined on a neighbourhood of $X_{0}$ in ID which is independent of $\Phi$ at $X_{0}$. Then $\bar{\Phi}^{l}(u)$ is involutive at $X_{0}$ if and only if $u$ is a relative integral of a characteristic system $\Delta^{l}\left(\mathfrak{B}_{\beta}\right)$ at $X_{0}$.

In this case, the characteristic module of $\Phi^{l}(u)$ at $X_{0}$ admits the primary decomposition in $L\left(X_{0}\right): N \cap\left\{\bigcap_{j \neq \beta} Q_{j}\left(X_{0}\right)\right\}$ where $N$ is either 
the total module or a $\mathfrak{P}_{\beta}\left(X_{0}\right)$-primary submodule containing $Q_{\beta}\left(X_{0}\right)$ such that $\mu\left(N ; L\left(X_{0}\right)\right)=\mu_{\beta}-1$.

Proof. Sufficiency: We prove that the four conditions (i)-(iv) of Theorem $\mathrm{A}$ in $\S 4$ are satisfied for $\Phi^{l}(u)$. Since $u$ is independent of $\Phi$ at $X_{0}$ and $u$ vanishes at $X_{0}$, it is clear that $X_{0}$ is an ordinary integral point of $\Phi^{l}(u)$. Hence the condition (i) is satisfied for $\Phi^{l}(u)$. Let us prove that the condition (iii) for $\Phi^{l}(u)$ is satisfied. We call in this proof a vector field $\sum_{i=1}^{n} \lambda_{i}(X) \partial / \partial x_{i}$ which assigns to each point in a subset of $I \Phi$ a vector of $c\left(\mathfrak{P}_{\beta}(X)\right)$ a characteristic vector field belonging to $c\left(\mathfrak{W}_{\beta}\right)$. Recalling the definition of characteristic systems and the argument in $\S 6$, we find no difficulty in showing that $u$ is a relative integral of $\Delta^{l}\left(\mathfrak{P}_{\beta}\right)$ at $X_{0}$ if and only if $u$ vanishes at $X_{0}$ and there is a neighbourhood $\mathscr{W}$ of $X_{0}$ in $I \Phi^{l}(u)$ such that

$$
\left\{\begin{array}{l}
\text { for any characteristic vector field } \sum_{i=1}^{n} \lambda_{i} \partial / \partial x_{i} \text { on } \mathscr{W} \\
\text { belonging to } c\left(\mathfrak{P}_{\beta}\right) \\
\sum_{i=1}^{n} \lambda_{i}\left(\frac{d u}{d x_{i}}+\sum_{\alpha=1}^{m} \sum_{i_{1} \leqq \cdots \leqq i_{l}} \psi_{\alpha}^{i_{1} \cdots i_{L} i} \frac{\partial u}{\partial p_{\alpha} i_{1} \cdots i_{l}}\right)=0 \\
\text { in consequence of (16) on } \mathscr{W} .
\end{array}\right.
$$

We put: $z(X)=\pi_{l}^{*} u \in L_{l}(X)$. Choosing W sufficiently small if necessary, we may assume that $u$ is independent of $\Phi$ at each point on $W$. This means that

$$
z(X) \notin M(X) \quad(X \in \mathscr{W}) .
$$

On the other hand, (23) implies that

$$
\left\{\begin{array}{l}
\text { for any characteristic vector field } \sum_{i=1}^{n} \lambda_{i} \partial / \partial x_{i} \text { on } \mathscr{W} \\
\text { belonging to } c\left(\mathfrak{P}_{\beta}\right) \\
\sum_{i=1}^{n} \lambda_{i}\left(\sum_{\alpha=1}^{m} \sum_{i_{1} \leq \cdots \leq i_{l}} \psi_{\alpha}^{i_{1} \cdots i_{l} i} \frac{\partial u}{\partial p_{\alpha}^{i_{1} \cdots i_{l}}}\right)=0 \\
\text { in consequence of (17) on } \mathscr{W} .
\end{array}\right.
$$

Since $\mathfrak{P}_{\beta}(X)$ is generated by $c\left(\mathfrak{P}_{\beta}(X)\right)$, it is readily seen that (25) means that 


$$
\mathfrak{P}_{\beta}(X) z(X) \subset M(X) \quad(X \in \mathscr{W}) .
$$

Applying Lemma 9 in $\S 8$, we conclude from (24) and (26) that $C_{X}$. $\left(\Phi^{l}(u)\right)$ is an involutive subspace of $Q_{\bar{X}}\left(J^{l-1}\right) \otimes T_{a}^{*}(\Re)$ for each $X \in \mathscr{W}$. Thus the condition (iii) is satisfied for $\Phi^{l}(u)$.

The condition (ii) is proved as follows. Obviously $g_{1}\left[C_{X}\left(\Phi^{l}(u)\right)\right]$ $=0$. Therefore, in virtue of Lemma 8 in $\S 8$, what we have just proved implies that the space $p C_{X}\left(\Phi^{l}(u)\right)$ has the same dimension as $C_{X}\left(\Phi^{l}(u)\right)$ for each $X \in \mathscr{W}$. Since $u$ is independent of $\Phi$ at $X \in \mathscr{W}$, the latter space has the dimension $\operatorname{dim} C_{X}(\Phi)-1$, which is constant on $\mathscr{W}$. Combining these, we know that the condition (ii) is satisfied for $\Phi^{l}(u)$.

It remains to prove that $\Phi^{l}(u)$ is $p$-closed at $X_{0}$. It suffices to show that the mapping $\rho_{l}{ }^{l+1}$ from $I\left\{p \Phi^{l}(u)\right\} \cap\left(\rho_{l}{ }^{l+1}\right)^{-1} \Psi$ to $W$ is surjective (§3), that is, for each $X \in \mathscr{W}$, the following system of linear equations in $p_{\alpha}{ }^{i_{1} \cdots i_{l+1}}$ actually possesses solutions:

$$
\begin{cases}\sum_{\alpha=1}^{m} \sum_{i_{1} \leqq \cdots \leqq i_{l}} \frac{\partial F}{\partial p_{\alpha}^{i_{1} \cdots i_{l}}} p_{\alpha}{ }^{i_{1} \cdots i_{l} i}+\frac{d F}{d x_{i}}=0 & \left\{\begin{array}{l}
i=1,2, \cdots, n, \\
F \in \Phi_{X}
\end{array}\right\}, \\
\sum_{\alpha=1}^{m} \sum_{i_{1} \leqq \cdots \leqq i_{i}} \frac{\partial u}{\partial p_{\alpha}^{i_{1} \cdots i_{l}}} p_{\alpha}^{i_{1} \cdots i_{l} i}+\frac{d u}{d x_{i}}=0 & (i=1,2, \cdots, n),\end{cases}
$$

the functions being supposed to denote their values at $X$. Since $c\left(\mathfrak{H}_{\beta}\right.$. $(X))$ is exactly the set of linear forms $\xi=\sum_{i=1}^{n} \lambda_{i} \partial / \partial x_{i}$ such that $\xi z(X)$ $\in M(X)$, it follows from (23) that (27) has solutions. Thus we have proved that the four conditions of Theorem A are satisfied for $\Phi^{l}(u)$, and hence $\Phi^{l}(u)$ is involutive at $X_{0}$.

Necessity: Suppose conversely that $\Phi^{l}(u)$ is involutive at $X_{0}$. There is a neighbourhood $W$ of $X_{0}$ in $I \Phi^{l}(u)$ such that $\Phi^{l}(u)$ is involutive at each point $X$ on $\mathscr{W}$ and its characters remains constant. On account of Theorem A, $C_{X}\left(\Phi^{l}(u)\right)$ is an involuitve subspace of $Q_{\bar{X}}\left(J^{l-1}\right) \otimes T_{a}{ }^{*}(N)$ for each $X \in \mathscr{W}$. Since $g_{1}\left[C_{X}\left(\Phi^{l}(u)\right)\right]=0$, we can apply Proposition 4 in $\S 7$; we know that there is a field of prime ideals $\mathfrak{\beta}_{\beta}(X)$ on $\Psi$ such that $C_{X}\left(\Phi^{l}(u)\right)$ contains $C\left(\mathfrak{F}_{\beta}(X)\right)$ for each $X \in \mathscr{W}$. Bearing in mind the assumption (a) in $(\mathrm{H}-2)$ and recalling the definition of the latter space (cf. $\S \S 6$ and 8), we conclude that (26) holds, and hence (25) holds. Now $\Phi^{l}(u)$ is $p$-closed at $X_{0}$, and hence we may assume that the mapping $\rho_{l}^{l+1}$ from $I\left\{p \Phi^{l}(u)\right\} \cap\left(\rho_{l}{ }^{l+1}\right)^{-1} \mathscr{W}$ to $\mathscr{W}$ is surjective; that 
is, system (27) actually admits solutions. Combining (25) with this fact, we conclude that (23) is valid. This means that $u$ is a relative integral of $\Delta^{l}\left(\mathfrak{F}_{\beta}\right)$ at $X_{0}$. The proof of Necessity is thus complete.

The characteristic module of $\Phi^{l}(u)$ at $X_{0}$ is nothing else than the module associated with the involutive subspace $C_{X}\left(\Phi^{l}(u)\right)$, which is of codimension 1 in $C_{X}(\Phi)$. Therefore the last assertion follows immediately from Proposition 5 in $\S 8$.

Q.E.D.

Proposition 7 can be generalized as follows.

Theorem V. Suppose that $\Phi$ satisfies the conditions $(\mathrm{H}-1)$ and $(\mathrm{H}-2)$. Let $X_{0}^{q}$ be a point of $I\left(p^{q-l} \Phi\right) \cap\left(\rho_{l}^{q}\right)^{-1} X_{0}(q \geqq l)$, and $u$ be a function of $q$-jets defined on a neighbourhood of $X_{0}^{q}$ which is independent of $\Phi$ at $X_{0}^{q}$. Then in order that $\Phi^{q}(u)$ is involutive at $X_{0}^{q}$, it is necessary and sufficient that $u$ is a relative integral of a characteristic system $\Delta^{q}\left(\mathfrak{P}_{\beta}\right)$ at $X_{0}^{q}$.

In this case, the characteristic module of $\Phi^{q}(u)$ at $X_{0}^{q}$ admits the primary decomposition in $L\left(X_{0}\right): N \cap\left\{\bigcap_{j \neq \beta} Q_{j}\left(X_{0}\right)\right\}$ where $N$ is either the total module or a $\mathfrak{P}_{\beta}\left(X_{0}\right)$-primary submodule containing $Q_{\beta}$. $\left(X_{0}\right)$ such that $\mu\left(N ; L\left(X_{0}\right)\right)=\mu_{\beta}-1$.

Proof. As we pointed out in $\S 7$, the prolonged system $p^{q-l} \Phi$ is involutive at $X_{0}^{q}$ and its characteristic module at $X_{0}^{q}$ is nothing else but that of $\Phi$ at $X_{0}$. Hence it is immediately seen that the conditions $(\mathrm{H}-1)$ and $(\mathrm{H}-2)$ are satisfied for $p^{q-l} \Phi$. Moreover, by the very definition, the characteristic systems of order $q$ of $\Phi$ are those (of order $q$ ) of $\rho^{q-l} \Phi$. Applying Proposition 7, we have at once the desired result.

Q.E.D.

Let us next try to construct involutive systems by adjoining to $\Phi$ several equations. Let $X^{q_{1}}, \cdots, Y^{q_{r}}$ be a point of $J^{q_{1}}, \cdots$, a point of $J^{q_{r}}$ respectively such that $\rho_{l}^{q_{i}} X^{q_{i}}=X_{0}$ and $\rho_{q_{j}}^{q_{i}} X^{q_{i}}=X^{q_{j}}$ for any pair $\left\{q_{i}, q_{j}\right\}$ with $q_{i} \geqq q_{j}\left(q_{1}, \cdots, q_{r} \geqq l\right)$. Let $\widetilde{X}_{0}$ denote such the point of $J^{q}$ that $\rho_{q_{i}}^{q} \widetilde{X}_{0}$ $=X^{q_{i}}(1 \leqq i \leqq r)$, where $q=\max \left\{q_{i} ; 1 \leqq i \leqq r\right\}$. Let $S=\left\{u_{1}, \cdots, u_{r}\right\}$ be a 
system of $r$ functions where each $u_{i}$ is a function of $q_{i}$-jets on a neighbourhood of $X^{q_{i}}$. We may assume without oss of generality that the functions $u_{i}$ are arranged in such a manner that $q_{1} \leqq q_{2} \leqq \cdots \leqq q_{r}$. We say that $S$ is independent of $\Phi$ over $X_{0}$ if and only if $u_{i+1}$ is independent of $\Phi^{q_{i}}\left(u_{1}, \cdots, u_{i}\right)$ at $X^{q_{i+1}}$ for each $i=0,1, \cdots, r-1$.

Theorem VI. Suppose that $\Phi$ satisfies the conditions $(\mathrm{H}-1)$ and $(\mathrm{H}-2)$, and that $\widetilde{X}_{0} \in I\left(p^{q-l} \Phi\right)$. Let $S_{1}=\left\{u_{1}, \cdots, u_{r_{1}}\right\}, \cdots, S_{s}=\left\{u_{r_{s-1}+1}, \cdots, u_{r}\right\}$ be $s$ systems of functions where each $u_{i}$ is a function of $q_{i}$-jets defined on a neighbourhood of $X^{q_{i}}$. If each system $S_{\beta}$ is independent of $\Phi$ over $X_{0}(1 \leqq \beta \leqq s)$ and if every $u_{i} \in S_{\beta}$ is a relative integral of $\lrcorner^{q_{i}}\left(\mathfrak{P}_{\beta}\right)$ at $X^{q_{i}}$, then $\Phi^{q}\left(u_{1}, \cdots, u_{r}\right)$ is involutive at $\widetilde{X}_{0}$.

If furthermore $r_{1}=\mu_{1}, r_{2}-r_{1}=\mu_{2}, \cdots, r-r_{s-1}=\iota_{s}$, then the characteristic module of $\Phi^{q}\left(u_{1}, \cdots, u_{r}\right)$ at $\widetilde{X}_{0}$ admits the reduced primary decomposition $\bigcap_{j=s+1}^{\nu} Q_{j}\left(X_{0}\right)$ in $L\left(X_{0}\right)$.

Proof. We may assume that $q_{1}$ is the smallest one of the numbers $q_{i}$. By Theorem $\mathrm{V}, \Phi^{q_{1}}\left(u_{1}\right)$ is involutive at $X^{q_{1}}$, and its characteristic module at $X^{q_{1}}$ admits the primary decomposition $N_{1} \cap\left\{\bigcap_{j=2}^{\nu} Q_{j}\left(X_{0}\right)\right\}$ where $N_{1}$ is either the total module or a $\mathfrak{B}_{1}\left(X_{0}\right)$-primary submodule such that $\mu\left(N_{1} ; L\left(X_{0}\right)\right)=\mu_{1}-1$. We readily see that the conditions $(\mathrm{H}-1)$ and $(\mathrm{H}-2)$ are satisfied for $\Phi^{q_{1}}\left(u_{1}\right)$ at $X^{q_{1}}$. Let $q_{k}$ be the smallest one of the numbers $q_{i}$ except $q_{1}$. For brevity we write $h=q_{k}$. When $u_{k} \in S_{1}$, it follows from the assumptions that $u_{k}$ is independent of $\Phi^{q_{1}}\left(u_{1}\right)$ at $X^{h}$. Suppose $u_{k} \in S_{\beta}$ with $\beta \neq 1$. We set $z_{k}(X)=\pi_{h}^{*} u \in L_{h}(X)$. The assumption that $u_{k}$ is a relative integral of $\Delta\left(\mathfrak{P}_{\beta}\right)$ independent of $\Phi$ at $X^{h}$ implies that $z_{k}\left(X_{0}\right) \oplus M_{l}\left(X_{0}\right)$ and $\mathfrak{\beta}_{\beta}\left(X_{0}\right) \quad z_{k}\left(X_{0}\right) \subset M\left(X_{0}\right) \quad$ (cf. the discussion in the proof of Proposition 7, in particular, (24) and (26)). By the same reasoning as in the proof of Proposition 6 in $\S 8$, we deduce from these that $z_{k}\left(X_{0}\right) \notin Q_{\beta}\left(X_{0}\right)$ and $z_{k}\left(X_{0}\right) \in \bigcap_{j \neq \beta} Q_{j}\left(X_{0}\right)$. It follows that $z_{k}$. $\left(X_{0}\right)$ does not belong to the characteristic module of $\Phi^{q_{1}}\left(u_{1}\right)$ at $X^{q_{1}}$. This means that $u_{k}$ is independent of $\Phi^{q_{1}}\left(u_{1}\right)$ at $X^{h}$. Thus, in any case, we can apply Theorem V. Hence we know that $\Phi^{h}\left(u_{1}, u_{2}\right)$ is involutive at $X^{h}$ and its characteristic module at $X^{h}$ is given by $N_{1}^{\prime} \cap\left\{\bigcap_{j \neq 1} Q_{j}\left(X_{0}\right)\right\}$ or 
$N_{1} \cap N_{\beta} \cap\left\{\bigcap_{j \neq 1, \beta} Q_{j}\left(X_{0}\right)\right\}$ according as $\iota_{k} \in S_{1}$ or $u_{k} \in S_{\beta}$, where the $N$ 's are primary submodules such that $\mu\left(N_{1}^{\prime} ; L\left(X_{0}\right)\right)=\mu_{1}-2, \mu\left(N_{\beta} ; L\left(X_{0}\right)\right)=\mu_{\beta}$ -1 . Proceeding step by step in the same way, we can complete the proof of the assertion that $\Phi^{q}\left(u_{1}, \cdots, u_{r}\right)$ is involutive at $\widetilde{X}_{0}$. It also follows that its characteristic module at $\widetilde{X}_{0}$ is given by $\left\{\bigcap_{j=1}^{s} Q_{j}{ }^{\prime}\right\} \cap\left\{\bigcap_{j=s+1}^{n} Q_{j}\right.$. $\left.\left(X_{0}\right)\right\}$ where the $Q_{j}{ }^{\prime}$ are primary sumbodules of $L\left(X_{0}\right)$ such that $\mu\left(Q_{j}{ }^{\prime}\right.$; $\left.L\left(X_{0}\right)\right)=\mu_{j}-\left(r_{j}-r_{j-1}\right) \quad(1 \leqq j \leqq s)$ where $r_{s}=r, r_{0}=0$.

Suppose $r_{j}-r_{j-1}=\mu_{j}(1 \leqq j \leqq s)$. Then $\mu\left(Q_{j}{ }^{\prime} ; L\left(X_{0}\right)\right)=0$, that is, the codimension of $Q_{j}{ }^{\prime} \cap L_{h}\left(X_{0}\right)$ in $L_{h}\left(X_{0}\right)$ equals zero for large $h ; Q_{j}{ }^{\prime} \cap L_{h}$ $\left(X_{0}\right)=L_{h}\left(X_{0}\right)$ (cf. the definition of multiplicities in $\S 1$ ). Since $Q_{j}^{\prime}$ is primary in $L\left(X_{0}\right), H\left(Q_{j}{ }^{\prime}\right)=Q_{j}{ }^{\prime}$ (see the beginning of $\S 3$ ). Combining these, we conclude that the submodules $Q_{j}{ }^{\prime}$ must be the total module. Consequently the last assertion is also valid.

Q.E.D,

\section{§ 10. The Method of Integration}

Suppose that $\mathscr{D}$ is involutive at $X_{0}$ and the characters $s_{2}\left(X_{0}\right), \cdots$, $s_{n}\left(X_{0}\right)$ vanish. As usual, we identify a solution of $\Phi$ with the corresponding $n$-dimensional integral manifold of $\Sigma(\Phi)$ in the space $J^{l}$. By a $p$ dimensional manifold in $J^{l}$, we mean all along such a manifold $\mathfrak{M}_{p}$ that $\left(\rho_{-1}^{l}\right)_{*} T_{X}\left(\mathfrak{M}_{p}\right)$ is of dimension $p$ for each point $X$ on $\mathfrak{M}_{p}$. An integral curve $\mathfrak{\Im}$ of $\Sigma(\Phi)$ is said to be non-characteristic if and only if no spaces $c\left(\mathfrak{F}_{j}(X)\right)$ contain $\left(\rho_{-1}^{l}\right)_{*} T_{X}(\mathfrak{\Im})$ for each $X \in \mathfrak{\Im}$. By the CartanKähler theorem (cf. Kähler [8], p. 26), there exists a unique analytic $n$ dimensional integral manifold of $\Sigma(\Phi)$ passing through a given non-characteristic integral curve. Thus the following Cauchy's problem possesses a unique solution: "Find an $n$-dimensional integral manifold of $\Sigma(\Phi)$ passing through a given non-characteristic integral curve." Throughout this section, by Cauchy's problem for $\Phi$ we mean the one just explained. The problem to engage our attention is the following one arisen from the classical method of integration: To reduce the solution of Cauchy's problem for $\Phi$ to the integration of a completely integrable Pfaffian system. (Refer to Goursat [6] and Forsyth [5] for the classical theory of integration. See also Kakié [9-10] for its recent development.)

Suppose $s_{1}\left(X_{0}\right)=0$. Then $\Phi$ is completely integrable at $X_{0}$; there 
passes a unique $n$-dimensional integral manifold of $\Sigma(\Phi)$ passing through a given integral point sufficiently near $X_{0}$. This manifold can be obtained by integrating the system $\Sigma(\Phi)$ which is completely integrable at $X_{0}$. We shall exclude this trivial case in the following discussion.

The vanishing of $s_{1}\left(X_{0}\right)$ means that the characteristic module $M\left(X_{0}\right)$ of $\Phi$ at $X_{0}$ coincides with the total module; $M\left(X_{0}\right)$ is a proper submodule of $L\left(X_{0}\right)$ if and only if $s_{1}\left(X_{0}\right)$ is positive. It is, however, the number $\nu$ of the components in the reduced primary decomposition of $M\left(X_{0}\right)$ which is of importance in treating our problem.

Suppose that $\Phi$ satisfies the conditions $(\mathrm{H}-1)$ and $(\mathrm{H}-2)$. Let us distinguish the following two cases.

$\left.1^{0}\right)$ The case when $\nu=1$ : By Theorem IV in $\S 6$, the differential system $\Sigma(\Phi)$ admits Cauchy-Cartan characteristics of dimension $n-1$. Moreover its Cartan characteristic system is given by the only one Monge characteristic system of order $l$ of $\Phi$. Let $\widetilde{\Im}$ be a non-characteristic integral curve of $\Sigma(\Phi)$. The one-parameter family of $(n-1)$-dimensional Cauchy-Cartan characteristic manifolds each of which passes through a point of $\mathfrak{\Im}$ generates an $n$-dimensional integral manifold $\mathfrak{M}_{n}$ of $\Sigma(\Phi)$ (cf. Cartan [4], Chap. III, Goursat [7], § 66). Since the Cartan characteristic system is completely integrable (Cartan [4], Chap. III), the manifold $\mathfrak{M}_{n}$ is obtained by integrating a completely integrable Pfaffian system. This fact may be stated as follows:

"If $\nu=1$, then the solution of Cauchy's problem for $\Phi$ can be reduced to the integration of a completely integrable Pfaffian system."

$2^{0}$ ) The case when $\nu>1$ : It is this case that we must consider to establish a method of integration. Let $\Im$ be a non-characteristic integral curve of $\Sigma(\Phi)$. The curve $\mathfrak{\Im}$ can be uniquely prolonged to a curve $\Im^{q}$ in $J^{q}$ in such a manner that $\mathfrak{\Im}^{q}$ is an integral curve of $\Sigma\left(p^{q-l} \Phi\right)$ and that $\rho_{l}^{q} \mathfrak{\Im}^{q}=\mathfrak{\Im}(q \geqq l)$.

Let $X^{q_{1}}, \cdots, X^{q_{\nu-1}}$ be a $q_{1}$-jet on $I\left(p^{q_{1}-l} \Phi\right), \cdots$, a $q_{\nu-1}$-jet on $I\left(p^{q_{\nu-1}-l} \Phi\right)$ respectively such that $\rho_{l}^{q_{i}} X^{q_{i}}=X_{0}$ and $\rho_{q_{j}}^{q_{i}} X^{q_{i}}=X^{q_{j}}$ for any pair $\left\{q_{i}, q_{j}\right\}$ with $q_{i} \geqq q_{j} \quad\left(q_{1}, \cdots, q_{\nu-1} \geqq l\right)$. We denote by $\widetilde{X}_{0}$ the $q$-jet on $I\left(p^{q-l} \Phi\right)$ determined by $\rho_{q_{i}}^{q} \widetilde{X}_{0}=X^{q_{i}}(1 \leqq i \leqq \nu-1)$, where $q=\max \left\{q_{i} ; 1 \leqq i \leqq \nu-1\right\}$. Let $\Delta\left(\mathfrak{P}_{1}\right), \cdots, \Delta\left(\mathfrak{P}_{\nu-1}\right)$ be $\nu-1$ distinct Monge characteristic systems of $\Phi$ over a neighbourhood of $X_{0}$ in $I \Phi$. 
Our method of integration is stated as follows:

“If each $\mathcal{S}\left(\mathfrak{F}_{\beta}\right)$ possesses $\mu_{\beta}+1$ functionally independent integrals of order $\leqq q_{\beta}$ defined on a neighbourhood of $X^{q_{\beta}}$ such that $\mu_{\beta}$ functions of them form a system independent of $\Phi$ over $X_{0}(1 \leqq \beta \leqq \nu-1)$, then for any non-characteristic curve $\mathfrak{\Im}$ such that $\mathfrak{\Im}^{q}$ passes sufficiently near $\widetilde{X}_{0}$, Cauchy's problem for $\Phi$ with initial curve $\Im$ can be solved by integrating a completely integrable Pfaffian system."

Applying Theorem VI in $\S 9$, we can construct a new involutive system admitting $\mathfrak{\Im}^{q}$ as an integral curve whose solutions are those of $\Phi$ and to which the method of integration stated in $1^{0}$ can be applied. The solution is obtained by applying to the new involutive system the method explained in $1^{0}$. The proof is carried out quite easily in just the same way as in the previous case (see Kakie [9], §7), and hence we omit the detail.

Some remarks concerning our method of integration are found in the last section of our previous work [9].

\section{Bibliography}

[1] Bourbaki, N., Éléments de mathématique, Algèbre commutative, Chap. IV, Hermann, Paris, 1961.

[2] Cartan, E., Sur l'intégration des systèmes d'équations aux différentielles totales, Ann. Sci. École Norm. Sup. $3^{\mathbf{e}}$ série 18 (1901), 241-311.

[3] —- Leçons sur les invariants intégraux, Hermann, Paris, 1922.

[4] - Les systèmes différentiels extérieurs et leurs applications géométriques, Hermann, Paris, 1945.

[5] Forsyth, A. R., Theory of Differential Equations, Part IV, Vol. VI, Cambridge Univ. Press, London 1906.

[6] Goursat, E., Leçons sur l'intégration des équations aux dérivées partielles du second ordre à deux variables indépendantes, Tom. II, Hermann, Paris, 1898.

[7] —_, Leşons sur le problème de Pfaff, Hermann, Paris, 1922.

[8] Kähler, E., Einführung in die Theorie der Systeme von Differentialgleichungen, Teubner, Leipzig. 1934.

[9] Kakié, K., On involutive systems of partial differential equations in two independent variables, J. Fac. Sci. Univ. Tokyo, Sect. IA, 21 (1974), 405-433.

[10] - On involutive systems of partial differential equations whose characters of order more than one vanish, Proc. Japan Acad., 51 (1975), 265-269.

[11] Kuranishi, M., On E. Cartan's prolongation theorem of exterior differential systems, Amer. J. Math., 79 (1957), 1-47.

[12] - - Lectures on involutive systems of partial differential equations, Pub. Soc. Mat. São Paulo, 1967.

[13] Matsuda, M., Cartan-Kuranishi's prolongation of differential systems combined with that of Lagrange and Jacobi, Publ. RIMS, Kyoto Univ., 3 (1967), 69-84. 
[14] - On involutive systems of partial differential equations, Sūgaku, 21 (1969), 161-177 (in Japanese).

[15] - Generalized Pfaff's problem, J. Fac. Sci. Univ. Tokyo, Sect. IA, 19 (1972), 231-242.

[16] Matsushima, Y., On a theorem concerning the prolongation of a differential system, Nagoya Math. J., 6 (1953), 1-16.

[17] Serre, J.-P., Algèbre Locale, Multiplicités, Lecture Notes in Mathematics, 11 Springer, Berlin, 1965.

[18] Zariski, O. and Samuel, P., Commutative Algebra, Vol. II, Van Nostrand, New York, 1960. 